Reviews and perspectives

\title{
Bilingual language lateralization: A meta-analytic tale of two hemispheres ${ }^{\text {is }}$
}

\author{
Rachel Hull*, Jyotsna Vaid \\ Department of Psychology, Texas A\&M University, College Station, TX 77843-4235, USA
}

Received 4 September 2006; received in revised form 16 February 2007; accepted 1 March 2007

Available online 7 March 2007

\begin{abstract}
Two meta-analyses of 66 behavioral studies examined variables influencing functional cerebral lateralization of each language of brain-intact bilingual adults. Functional lateralization was found to be primarily influenced by age of onset of bilingualism: bilinguals who acquired both languages by 6 years of age showed bilateral hemispheric involvement for both languages, whereas those who acquired their second language after age 6 showed left hemisphere dominance for both languages. Moreover, among late bilinguals, left hemisphere involvement was found to be greater for those less proficient in their second language, those whose second language was English, and for studies involving dichotic listening paradigms; early bilinguals instead showed bilateral involvement in every condition. Implications of the observed differences in lateralization between early and late bilinguals are explored for existing theories of bilingualism and for neurocognitive models of brain functional organization of language. (C) 2007 Elsevier Ltd. All rights reserved.
\end{abstract}

Keywords: Brain; Language experience; Laterality; Second language; Cerebral asymmetry; Bilingual

\section{Contents}

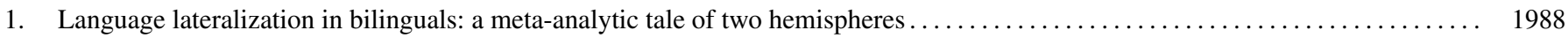

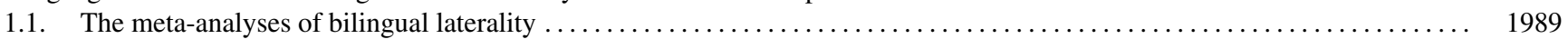

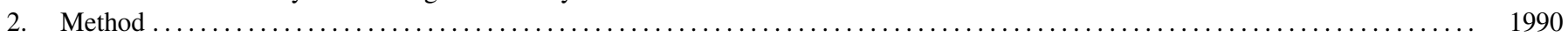

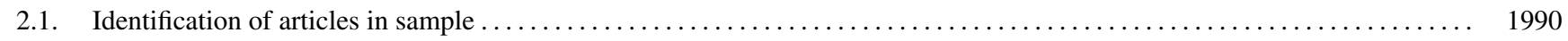

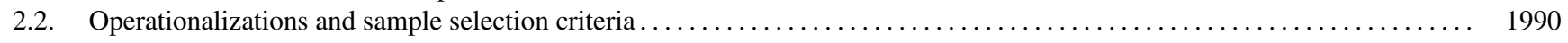

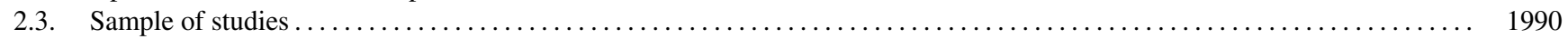

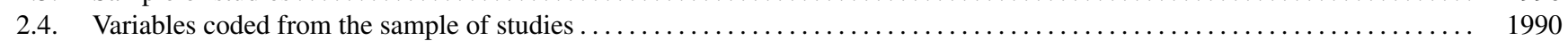

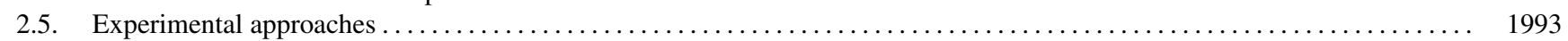

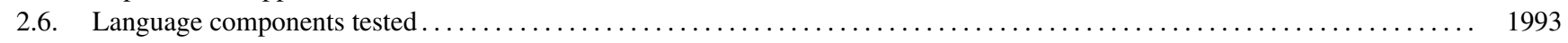

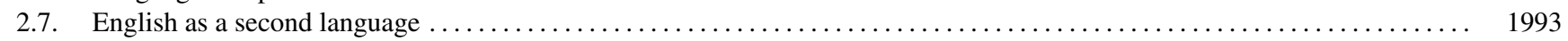

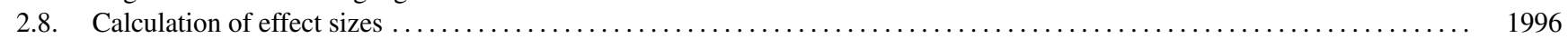

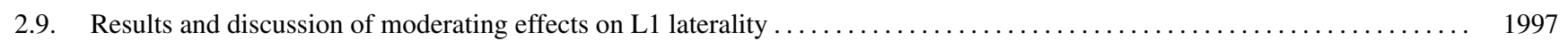

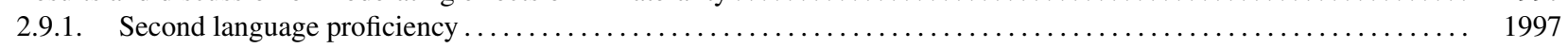

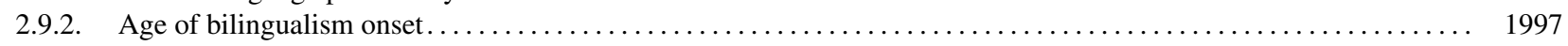

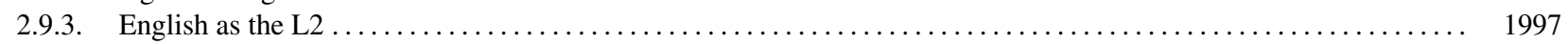

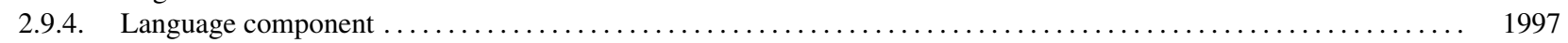

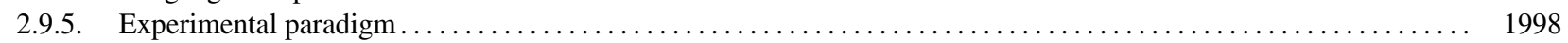

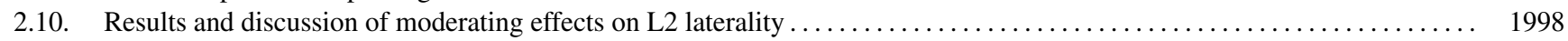

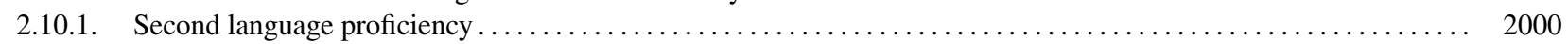

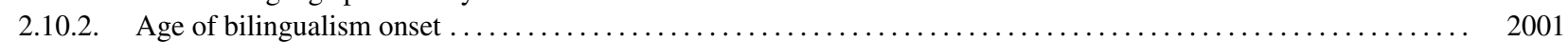

\footnotetext{
is This research constituted part of the first author's dissertation. Portions of this research were presented at the 4th International Symposium on Bilingualism held in Tempe, AZ, 2003.

* Corresponding author. Tel.: +1 979224 0212; fax: +1 9798454727.

E-mail address: rhull@tamu.edu (R. Hull).
} 


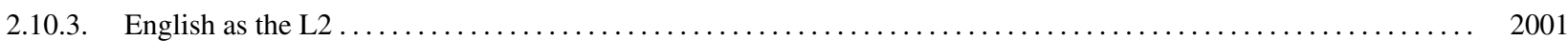

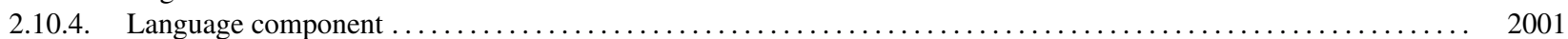

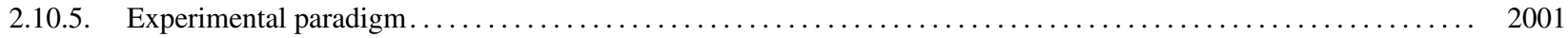

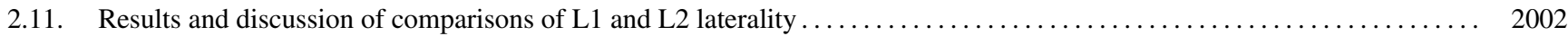

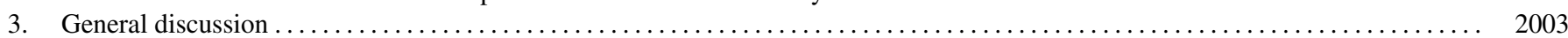

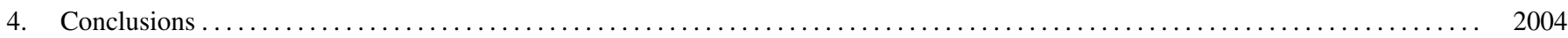

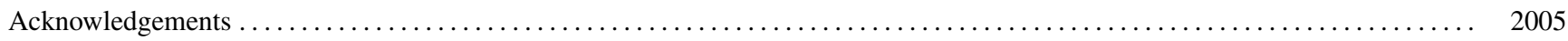

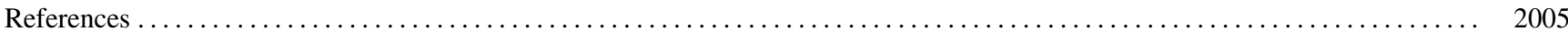

\section{Language lateralization in bilinguals: a meta-analytic tale of two hemispheres}

The steady rise of studies investigating the neural bases of language organization in users of more than one language underscores a growing recognition that bi- or multilingualism is a pervasive characteristic of language use and one that models of functional organization of language need to address to understand the full range of actual language experience. Brain functioning is known to be sensitive to variations in early sensory experience (e.g., Neville et al., 1997). Bilingualism offers a unique opportunity to explore the potential influence of variation in early language experience on the shaping of brain function and structure and its potential for plasticity (see Golestani \& Zatorre, 2004; Mechelli et al., 2004). Although more than a hundred behavioral bilingual laterality studies (see Hull \& Vaid, 2006; Vaid \& Hall, 1991, for reviews) and several dozen functional neuroimaging studies with bilinguals have been conducted (see Abutalebi, Cappa, \& Perani, 2005; Goral, Levy, \& Obler, 2002; Vaid \& Hull, 2002, for reviews), their findings are only beginning to be systematically quantified (see Hull \& Vaid, 2006; Indefrey, 2006) and their implications explored for the ongoing debate about a sensitive period for language acquisition (see Bialystok, 2001; Birdsong \& Molis, 2001; Johnson \& Newport, 1989; Sebastian-Galles \& Bosch, 2005).

The central aim of the present research was to systematically examine and quantify, through meta-analysis, outcomes from the behavioral bilingual laterality literature in order to test two key hypotheses that have laid the groundwork for much past and present research on the functional organization of language in the bilingual brain: the Age of Language Acquisition Hypothesis (henceforth, the Age Hypothesis; Genesee et al., 1978; Vaid, 1983; Vaid \& Genesee, 1980) and the Stage of Language Acquisition Hypothesis (henceforth, the Stage Hypothesis; Albert \& Obler, 1978; Galloway \& Krashen, 1980; Obler, 1981; Schneiderman, 1983; Vaid, 1983). The effects of second language acquisition age and proficiency have been extensively studied and debated within the second language acquisition literature (e.g., Birdsong, 2005; DeKeyser \& LarsonHall, 2005; see also Franceschini, Zappatore, \& Nitsch, 2003). For the present purposes, it should be noted that the Age and Stage Hypotheses were operationalized to allow for a level of specificity deemed necessary to allow formal testing, although this level of specificity was not always present in the original formulations in the bilingual laterality studies that first explored these variables.
The Age Hypothesis predicts that language lateralization will depend on the temporal proximity of acquisition, such that early, near simultaneous acquisition of the two languages will yield a different pattern of lateralization than late, successive acquisition of the second language (L2) relative to the first language (L1). The rationale for the Age Hypothesis rests on cognitive and brain maturational differences. For example, it is known that neither the cerebral cortex nor the corpus callosum is fully developed until the age of 5 or 6 years (or even later, see Lenneberg, 1969) and that myelination of neural pathways is not established until that time and even later (see discussion in Long, 1990). These observations, coupled with socio-cognitive differences accompanying early versus late L2 acquisition, have motivated the search for differences in the neural and/or functional architectures supporting language in early versus late bilinguals (e.g., Fabbro, 2001; Perani et al., 1996; Vaid \& Hull, 2002; Vaid, in press).

The Stage Hypothesis, by contrast, emphasizes relative proficiency in the $\mathrm{L} 2$ as the determining factor in functional language laterality. It predicts that increasing proficiency in the L2 will be accompanied by a shift from an initial reliance on discourse level pragmatic cues thought to be mediated by the right hemisphere ( $\mathrm{RH})$ to subsequent left hemisphere ( $\mathrm{LH})$ dominance as syntactic and phonological rules become more automatized.

It should be noted that neither of these central hypotheses nor any of the empirical studies in the present sample isolated length of language use as a variable, and thus it could not be specifically tested in the present research. However, because bilinguals who learned two languages from birth will have necessarily used both languages longer than those who acquired an L2 later in life, regardless of proficiency, we acknowledge that length of language use is an important variable to be considered in future research.

Taken as a whole, findings from bilingual laterality studies have presented an array of patterns of lateralization that have not lent themselves to unqualified support for one hypothesis or another. Indeed, some scholars have taken conflicting outcomes in the bilingual laterality literature as support for their belief that behavioral laterality measures lack reliability and validity (see Paradis, 2003). However, this criticism appears to be unfounded, since evidence for the reliability and validity of laterality measures can be marshaled. For example, with regard to test-retest reliability, Segalowitz et al. have shown that a majority of participants showed stable lateralization of function across behavioral sessions (especially when the interval between sessions was relatively short) and suggested that the minority of 
"deviant" individuals might have been actively pursuing different strategies to improve their performance or to make the task more interesting (Segalowitz, 1986; see also Segalowitz \& Bryden, 1983).

With regard to validity, dichotic listening, tachistoscopic viewing, and dual task paradigms have each been validated against clinical tests, typically the sodium amytal or Wada test (Kosaka, Hiscock, Strauss, Wada, \& Purves, 1993; Segalowitz, 1986). For example, Kosaka et al. found that there was greater right hand than left hand tapping interference during a concurrent linguistic task among right handed patients whose amytal test indicated left hemisphere speech dominance; similarly, for right handed patients with right hemisphere speech dominance, greater left hand tapping interference was found. Dichotic listening has also been validated against electroencephalogram activity recorded during verbal tasks (Davidson \& Hugdahl, 1996).

In light of evidence that behavioral laterality measures are reasonably valid and reliable, a more likely source of inconsistent findings observed across bilingual laterality studies may be variation in subject variables, such as degree of proficiency or age of onset of bilingualism. Characteristics of the languages of the bilinguals, as well as processing demands of the task or paradigm may also be potential sources of variability across studies (Abutalebi et al., 2005; Obler, 1981; Obler, Zatorre, Galloway, \& Vaid, 1982; Sebastian-Galles \& Bosch, 2005; Soares, 1984; Zatorre, 1989; see also Rosenthal \& DiMatteo, 2001).

Given the number of dimensions on which studies in the bilingual laterality literature have differed, it is not surprising that traditional narrative reviews of this literature have found it difficult to arrive at definitive conclusions. A meta-analytic approach, as used in the present research, allows one to systematically disentangle the complex outcomes in a literature and to identify reliable findings, thus making it possible to arrive at generalizations. In a recent meta-analysis, Hull and Vaid (2006) examined bilingual functional lateralization based on studies that directly compared monolinguals and bilinguals and found that monolinguals and late bilinguals were reliably LH dominant across language tasks regardless of proficiency, whereas early bilinguals showed reliable bilateral hemispheric involvement (comparable differences between early and late bilinguals were also reported in an earlier meta-analysis by Vaid \& Hall, 1991). Moreover, LH participation was found to be greater in studies that used the dichotic listening task relative to other testing paradigms, regardless of language experience or proficiency (Hull \& Vaid, 2006). Taken together, this evidence suggests that the primary predictor of functional language lateralization in adulthood (at least for native languages) is whether an individual learned one versus two languages during early development.

Whereas the evidence discussed so far indicates that behavioral measures of language laterality can be used to demonstrate reliable differences in functional brain lateralization among bilinguals with different language experience histories, it remains to be determined whether differences in lateralization for both languages will obtain between bilingual groups differing in age of onset of bilingualism, and/or in language proficiency, and whether differences in lateralization between the two languages will be obtained within each bilingual group. The present meta-analysis addresses these questions and also tests for the effects of the nature of language, the language component tested, and the nature of the laterality paradigm using a much larger corpus of bilingual laterality studies than has been considered to date.

\subsection{The meta-analyses of bilingual laterality}

Because meta-analytic outcomes must be reported in statistically similar forms in order to be meaningfully aggregated (Lipsey \& Wilson, 2001), each study included in the present research provided results from which an effect size statistic could be calculated from group means and standard deviations of performance on identical language tasks. Moreover, to satisfy the requirement of conceptual comparability in terms of constructs and the relationships between variables (Lipsey and Wilson), all studies conceptualized hemispheric mediation of language in terms of overt responses (whether vocal or manual) to visual or auditory language input initially directed to one hemisphere or the other. As such, all studies in the present data set used the relationship between active language performance and the hemisphere to which stimuli were presented to infer hemispheric participation on language tasks. In contrast, studies were excluded if they did not specifically report data from each of the two hemispheres during identical tasks, or if they used passive tasks or measures (e.g., watching words on a video screen while correlated brain activity was collected). Thus, neuroimaging data, including hemodynamic and electrophysiological measures, were excluded because they typically focus on a particular region of interest within a single hemisphere (e.g., Broca's area), with the goal of relating metabolic (or electrical) activity in the region of interest with a particular aspect of language processing compared to that during a different baseline condition, often without an overt behavioral response measure.

Once the domain of analysis was constrained to behavioral studies on the statistical and theoretical grounds just discussed, the present research was designed to evaluate the bilingual laterality literature in three ways. First, it assessed all available behavioral laterality studies to date (published and unpublished) that were carried out with bilingual participants. Second, it evaluated the effects of specific moderators, including age of bilingualism onset and L2 proficiency. Third, it separately considered and then compared laterality effects for L1 and L2.

In what follows, outcomes are reported from 2 meta-analyses of 66 empirical studies of the cerebral lateralization of language in bilinguals during L1 and/or L2 tasks. Whereas both languages of early bilinguals can be considered first languages, L1 was classified here according to self-report, author notation, or the language of regional prevalence. Separate meta-analyses were conducted for L1 and for L2; each examined the effects of age of bilingualism onset, L2 proficiency, experimental paradigm, English as the L2, and language component tested. After describing the selection criteria and data analysis, the separate and combined influences of these moderators are presented for L1 and for L2. Next, the results of direct comparisons of L1 and L2 
performance within the same bilingual individuals are presented and used to evaluate theories concerning brain organization for multiple languages. Finally, implications for theory and future behavioral and imaging research are discussed.

\section{Method}

\subsection{Identification of articles in sample}

The research domain for the present meta-analyses included all behavioral language laterality studies conducted and/or published through the end of September 2004. An exhaustive literature search for published and unpublished studies that assessed language lateralization in neurologically healthy bilinguals was conducted through electronic keyword searches of PsycINFO (1872-2004), ERIC (1966-2004), Linguistics and Language Behavior Abstracts (1973-2004), and Dissertation Abstracts International (1861-2004). The keywords used were bilingual ${ }^{*}+$ language, bilingual ${ }^{*}+$ linguistic $^{*}$, bilingual $^{*}+$ lateral $^{*}$,

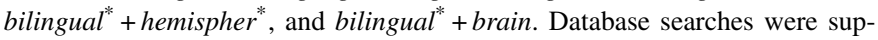
plemented by manual searches of the following periodicals dated from January 1998 through September 2004: Brain, Journal of Applied Psycholinguistics, Science, Journal of Memory and Language, Brain and Cognition, Language and Cognitive Processes, Psychological Science, and Journal of Phonetics. In addition, electronic cited-reference forward and author searches were used, as were manual reviews of the reference lists of included studies.

As recommended in the meta-analysis literature (e.g., Cooper \& Hedges, 1994; Rosenthal, 1991), unpublished and missing data were pursued through direct correspondence with knowledgeable bilingual laterality researchers. As a result, several unpublished studies included in this meta-analysis represented extensions of or follow-ups to previously published studies. The rationale for including such data was to reduce the potential for publication bias and to provide a more comprehensive sampling of the research literature (see Cooper \& Hedges, 1994; Rosenthal, 1991). Publication status (published or unpublished) was coded in each study, and comparisons revealed no significant differences among effect sizes drawn from published versus unpublished sources. All unpublished, non-public data used in the present research (denoted by superscript $d$ in Tables 1 and 2 ) will be maintained and provided to interested researchers upon request to the first author of the present article.

\subsection{Operationalizations and sample selection criteria}

The attributes used to define bilingualism and its subtypes have varied widely from study to study. Therefore, moderator divisions were clarified as follows:

- Bilinguals-individuals possessing a functional knowledge of at least two languages, although the degree of linguistic/communicative ability could vary across languages.

- Infant bilinguals-individuals who acquired both languages by the age of 6 years.

- Childhood bilinguals-individuals who acquired L2 after age 6 and before age 13 .

- Adult bilinguals-individuals who acquired L2 at or after the age of 13 .

- Proficient bilinguals -individuals whose language performance on standardized proficiency exams was reported at or above $85 \%$ accuracy, or who gave teacher- or self-ratings as "high" on proficiency, and/or had 5 or more years of formal study of the language.

- Nonproficient bilinguals-individuals who did not report meeting any of the above proficiency criteria.

The criteria for inclusion in the meta-analysis were as follows: published or unpublished studies of brain-intact bilinguals that assessed overt behavioral performance in response to visual or auditory language input initially directed to one hemisphere versus to the other. In addition, study inclusion required that age of bilingualism onset and stage of L2 proficiency was stated or inferable in the study itself or provided through personal communication with study authors. Fourteen of the 66 total studies (i.e., 21\%) included data obtained by personal communication. These studies are denoted by superscript $d$ in Tables 1 and 2 .
The criteria for excluding studies were: use of brain-damaged individuals, lack of uniformity in data collection conditions across the two languages, reliance on tasks or response measures in which participants' performance was not directly monitored but had to be inferred, (e.g., covert performance relative to a different baseline task), or lack of availability of appropriate quantitative data. In addition, data from users of sign language were excluded so as to provide homogeneity in language mode, particularly given suggestions that sign language use is associated with patterns of brain activity that differ from those associated with spoken languages (e.g., Neville et al., 1997).

\subsection{Sample of studies}

In all, 66 behavioral language laterality studies with bilinguals met the criteria for inclusion. Of these, 46 were published and 20 were unpublished. Further, 46 studies provided separate data for L1 and L2, 13 studies provided only L2 data, and 10 studies provided only data comparing L1 and L2. A total of 98 statistically independent effect sizes were generated for the synthesis of mean effect sizes for L1s (see Table 1) and 106 were generated for L2s (see Table 2).

\subsection{Variables coded from the sample of studies}

The meta-analyses used fixed effects computational models with a categorical model-fitting approach (Hedges \& Olkin, 1985) based on the goal of partitioning the individual and combined effects of a relatively large number of categorical variables identified in the literature. First, the main effects of L1 and L2 laterality were calculated. Next, each was separately modeled for each of the a priori moderator divisions in a fully hierarchical manner (Arthur, Bennett, \& Huffcutt, 2001; see also Hedges \& Becker, 1986; Hunter \& Schmidt, 1990; Voyer, 1996). Analyses were performed until effect size variance was homogeneous, at which point the mean effect size was taken to be an accurate estimate of the associated population, and sources of group differences were identified (Hunter \& Schmidt, 1990). However, higher level interactions were not reported when the majority of variance was already explained by lower-level interactions. In addition, higher level interactions were not reported when comparison cells contained five or fewer data points because reliability of outcomes is compromised in such cases (Arthur et al., 2001; Lipsey \& Wilson, 2001).

Finally, the main effect of L1 versus L2 laterality was computed from the mean effect size for L1 laterality across studies versus the mean effect size for L2 laterality across studies, where each study provided at most a single data point for each language. For the 10 studies where effect sizes for L1 and L2 could not be separately computed ${ }^{1}$, we calculated the mean effect size for the difference between L1 and L2 laterality within each study. Given that the two analysis strategies provided essentially identical outcomes, only the categorical models for the study-wide effect sizes were reported. All analyses in the present research synthesis were conducted using Johnson's (1993) DSTAT 1.10 software for the meta-analytic review of research literatures.

The categorical models used in the present research were: (a) age of L2 acquisition onset (early, childhood, adult), (b) stage of L2 acquisition (proficient, nonproficient), (c) experimental paradigm (visual preference, dichotic listening, dual task), (d) language component (single words, word-pair phonological, word-pair orthographic, word-pair semantic, word-pair syntactic, whole sentence), and (e) the nature of the L2 (English, other). Agreement was 92\% among three independent raters, and inconsistencies were resolved through discussion. Also coded were source type (published or unpublished), year of study completion/publication, gender composition of the sample, mean age of participants at testing, whether moderator values were explicitly stated or inferred, relatedness of L1 and L2 linguistic structures, and formal versus informal context of language use; none of these additional variables yielded significant effects and are not further discussed.

\footnotetext{
1 Albert and Obler (1978), Bergh (1986), Carroll (1980), Galloway and Scarcella (1982), Judd (1986), Kotik (1975), Lubow, Tsal, Mirkin, and Mazliah (1994), McClung (1981), Orbach (1967), Soares (1982), Soares and Grosjean (1981).
} 
Table 1

Data for bilingual L1 laterality

\begin{tabular}{|c|c|c|c|c|c|c|c|c|}
\hline Study author(s), year & L1-L2 & Group $n$ & $d$ & $95 \% \mathrm{CI}$ & $\begin{array}{l}\text { Age of L2 } \\
\text { onset }\end{array}$ & L2 proficiency & Language component & $\begin{array}{l}\text { Experimental } \\
\text { paradigm }\end{array}$ \\
\hline Bentin $(1981)^{\mathrm{a}, \mathrm{c}}$ & $\mathrm{He}-\mathrm{En}$ & 32 & 0.50 & $0.00,0.10$ & $\mathrm{C}$ & Nonproficient & 1 & $\mathrm{~V}$ \\
\hline Chengappa and Ray $(2002)^{\mathrm{d}}$ & Kann-En & 10 & 0.22 & $-0.66,1.10$ & $\mathrm{C}$ & Proficient & 1 & $\mathrm{~V}$ \\
\hline Endo, Shimizu, and Nakamura (1981a) ${ }^{a}$ & $\mathrm{Ka}-\mathrm{Ha}$ & 13 & 0.18 & $-0.59,0.95$ & A & Nonproficient & 1 & DT \\
\hline Fabbro (1992, Exp. 2) ${ }^{\mathrm{a}}$ & It-Ge & 3 & 0.75 & $-0.90,2.41$ & A & Proficient & 6 & DL \\
\hline \multicolumn{9}{|l|}{ Fabbro $\left(1992\right.$, Exp. 4) ${ }^{\mathrm{a}}$} \\
\hline Group 1 & It-Fri & 12 & 0.20 & $-0.60,1.00$ & I & Proficient & 6 & DT \\
\hline Group 2 & It-Fri & 12 & 0.07 & $-0.73,0.87$ & I & Proficient & 6 & DT \\
\hline Group 3 & It-Fri & 7 & 0.06 & $-0.99,1.11$ & I & Proficient & 6 & DT \\
\hline Fabbro, Gran, and Gran (1991) ${ }^{\mathrm{a}}$ & It-En & 36 & 0.17 & $-0.29,0.63$ & A & Proficient & 6 & DL \\
\hline Fabbro, Gran, Basso, and Bava (1990) & It-En & 14 & 0.02 & $-0.72,0.76$ & A & Proficient & 6 & DT \\
\hline \multicolumn{9}{|l|}{ Fabbro, Gran, and Bava (1988) } \\
\hline Group 1 & It-En & 12 & 0.87 & $0.03,1.71$ & A & Proficient & 1 & DL \\
\hline Group 2 & It-En & 12 & 1.10 & $0.24,1.95$ & A & Proficient & 1 & DL \\
\hline \multicolumn{9}{|l|}{ Furtado and Webster (1991) ${ }^{\mathrm{a}}$} \\
\hline Group 1 & En-Fr & 16 & -0.08 & $-0.78,0.61$ & I & Proficient & 6 & DT \\
\hline Group 2 & $\mathrm{En}-\mathrm{Fr}$ & 16 & 0.15 & $0.54,0.85$ & A & Proficient & 6 & DT \\
\hline Group 3 & Fr-En & 16 & -0.11 & $0.30,1.77$ & A & Proficient & 6 & DT \\
\hline \multicolumn{9}{|l|}{ Green $(1986)^{\mathrm{a}}$} \\
\hline Group 1 & En-Sp & 24 & -0.08 & $-0.64,0.49$ & A & Proficient & 6 & DT \\
\hline Group 2 & En-Sp & 24 & -0.20 & $-0.77,0.36$ & A & Nonproficient & 6 & DT \\
\hline Group 3 & En-Sp & 24 & -0.28 & $-0.85,0.29$ & A & Nonproficient & 6 & DT \\
\hline \multicolumn{9}{|c|}{ Green, Schweda-Nicholson, Vaid, White, and Steiner (1990) ${ }^{\mathrm{a}}$} \\
\hline Group 1 & Sp-En & 8 & 0.46 & $-0.54,1.45$ & $\mathrm{C}$ & Proficient & 6 & DT \\
\hline Group 2 & $\mathrm{Sp}-\mathrm{En}$ & 8 & 0.48 & $-0.52,1.47$ & $\mathrm{C}$ & Proficient & 6 & DT \\
\hline Group 3 & Sp-En & 8 & 0.49 & $-0.51,1.48$ & $\mathrm{C}$ & Proficient & 6 & DT \\
\hline Group 4 & Sp-En & 8 & 0.37 & $-0.61,1.36$ & $\mathrm{C}$ & Proficient & 6 & DT \\
\hline \multicolumn{9}{|l|}{ Hall and Lambert (1988) } \\
\hline Group 1 & $\mathrm{En}-\mathrm{Fr}$ & 16 & -0.12 & $-0.82,0.57$ & $\mathrm{C}$ & Proficient & 6 & DT \\
\hline Group 2 & $\mathrm{En}-\mathrm{Fr}$ & 16 & 0.26 & $-0.43,0.96$ & $\mathrm{C}$ & Proficient & 6 & DT \\
\hline Group 3 & $\mathrm{En}-\mathrm{Fr}$ & 16 & 0.20 & $-0.49,0.90$ & $\mathrm{C}$ & Nonproficient & 6 & DT \\
\hline Hoosain and Shiu (1989) & Ch-En & 28 & 0.82 & $0.28,1.37$ & $\mathrm{C}$ & Proficient & 1 & $\mathrm{~V}$ \\
\hline \multicolumn{9}{|l|}{ Ip and Hoosain (1993) $)^{\mathrm{a}, \mathrm{c}}$} \\
\hline Group 1 & Ch-En & 9 & 0.31 & $-0.62,1.24$ & $\mathrm{C}$ & Proficient & 1 & DL \\
\hline Group 2 & Ch-En & 9 & 0.12 & $-0.80,1.05$ & $\mathrm{C}$ & Proficient & 1 & DL \\
\hline $\operatorname{Jin}(1988)^{b, c}$ & $\mathrm{Ha}-\mathrm{En}-\mathrm{Ch}$ & 24 & 1.08 & $0.48,1.69$ & A & Proficient & 3 & DL \\
\hline \multicolumn{9}{|l|}{$\operatorname{Ke}(1992)^{a, c}$} \\
\hline Group 1 & $\mathrm{En}-\mathrm{Ch}$ & 28 & 0.20 & $-0.33,0.72$ & A & Proficient & 1 & DL \\
\hline Group 2 & $\mathrm{En}-\mathrm{Ch}$ & 29 & 0.77 & $0.24,1.31$ & A & Nonproficient & 1 & DL \\
\hline \multicolumn{9}{|l|}{ Mägiste (1989)a } \\
\hline Group 1 & $\mathrm{Ge}-\mathrm{Sw}$ & 9 & -0.22 & $-1.14,0.71$ & $\mathrm{C}$ & Proficient & 6 & $\mathrm{~V}$ \\
\hline Group 2 & $\mathrm{Ge}-\mathrm{Sw}$ & 9 & -0.14 & $-1.07,0.78$ & $\mathrm{C}$ & Proficient & 6 & $\mathrm{~V}$ \\
\hline \multicolumn{9}{|l|}{ Mägiste (1987) } \\
\hline Group 1 & $\mathrm{Ge}-\mathrm{Sw}$ & 10 & 0.46 & $-0.42,1.35$ & I & Proficient & 1 & $\mathrm{~V}$ \\
\hline Group 2 & $\mathrm{Ge}-\mathrm{Sw}$ & 10 & 0.57 & $-0.33,1.46$ & A & Proficient & 1 & $\mathrm{~V}$ \\
\hline Group 3 & Po-Sw & 14 & 0.70 & $-0.07,1.46$ & A & Nonproficient & 1 & $\mathrm{~V}$ \\
\hline Manga and Sanchez (1989) $)^{\mathrm{a}, \mathrm{c}}$ & En-Sp & 31 & 0.54 & $0.03,1.04$ & $\mathrm{C}$ & Proficient & 6 & DT \\
\hline $\operatorname{Rupp}(1980)^{\mathrm{b}}$ & Vi-En & 86 & 0.83 & $0.52,1.14$ & $\mathrm{C}$ & Nonproficient & 1 & DL \\
\hline Sakhuja $(1990)^{\mathrm{b}, \mathrm{c}}$ & Ur-En & 40 & 0.12 & $-0.13,0.38$ & I & Proficient & 4 & DT \\
\hline \multicolumn{9}{|l|}{ Sewell and Panou (1983) } \\
\hline Group 1 & En-Ge & 6 & 0.14 & $-0.99,1.28$ & I & Proficient & 1 & $\mathrm{~V}$ \\
\hline Group 2 & En-Ge & 6 & 0.21 & $-0.93,1.34$ & I & Proficient & 1 & $\mathrm{~V}$ \\
\hline Group 3 & $\mathrm{En}-\mathrm{Fr}$ & 6 & 0.45 & $-0.70,1.59$ & A & Proficient & 1 & $\mathrm{~V}$ \\
\hline Group 4 & $\mathrm{En}-\mathrm{Fr}$ & 6 & 0.22 & $-0.91,1.36$ & $\mathrm{~A}$ & Proficient & 1 & $\mathrm{~V}$ \\
\hline \multicolumn{9}{|l|}{ Shanon $(1982)^{\mathrm{a}}$} \\
\hline Group 1a & He-En & 8 & -0.02 & $-1.00,0.96$ & A & Proficient & 1 & $\mathrm{~V}$ \\
\hline
\end{tabular}


Table 1 (Continued)

\begin{tabular}{|c|c|c|c|c|c|c|c|c|}
\hline Study author(s), year & L1-L2 & Group $n$ & $d$ & $95 \% \mathrm{CI}$ & $\begin{array}{l}\text { Age of L2 } \\
\text { onset }\end{array}$ & L2 proficiency & Language component & $\begin{array}{l}\text { Experimental } \\
\text { paradigm }\end{array}$ \\
\hline Group 1b & He-En & 8 & -0.03 & $-1.01,0.95$ & A & Proficient & 1 & $\mathrm{~V}$ \\
\hline Group 2a & He-En & 8 & 0.99 & $-0.79,1.17$ & I & Proficient & 1 & V \\
\hline Group $2 b$ & He-En & 8 & 0.28 & $-0.70,1.27$ & I & Proficient & 1 & $\mathrm{~V}$ \\
\hline Group 3a & $\mathrm{En}-\mathrm{He}$ & 8 & 0.37 & $-0.62,1.36$ & A & Proficient & 1 & V \\
\hline Group 3b & $\mathrm{En}-\mathrm{He}$ & 8 & 0.31 & $-0.67,1.30$ & A & Proficient & 1 & $\mathrm{~V}$ \\
\hline \multicolumn{9}{|l|}{ Singh $(1990)^{\mathrm{a}, \mathrm{c}}$} \\
\hline Group 1 & Hi-En & 18 & -0.11 & $-0.77,0.54$ & I & Proficient & 6 & DT \\
\hline Group 2 & Hi-En & 18 & -0.27 & $-0.92,0.39$ & I & Proficient & 6 & DT \\
\hline Soares $(1984)^{\mathrm{a}}$ & Por-En & 16 & 1.73 & $0.92,2.55$ & A & Proficient & 6 & DT \\
\hline \multicolumn{9}{|c|}{ Spiller-Bosatra, Daro, Fabbro, and Bosatra $(1990)^{\mathrm{a}}$} \\
\hline Group 1 & It-Ge & 3 & -0.53 & $-2.16,1.09$ & I & Proficient & 1 & DL \\
\hline Group 2 & It-Ge & 5 & 0.28 & $-0.96,1.53$ & I & Proficient & 1 & DL \\
\hline Starck, Genesee, Lambert, and Seitz (1977) ${ }^{\mathrm{a}}$ & $\mathrm{En}-\mathrm{Fr}-\mathrm{He}$ & 24 & 0.74 & $0.16,1.33$ & I & Proficient & 1 & DL \\
\hline Thomas $(1987)^{\mathrm{b}, \mathrm{c}}$ & $\mathrm{Ch}-\mathrm{En}$ & 26 & 0.09 & $-0.46,0.63$ & A & Proficient & 1 & DL \\
\hline \multicolumn{9}{|l|}{ Vaid $(2003)^{\mathrm{d}}$} \\
\hline Group 1 & Hi-En & 16 & -0.26 & $-0.96,0.43$ & $\mathrm{C}$ & Proficient & 4 & $\mathrm{~V}$ \\
\hline Group 2 & $\mathrm{Ur}-\mathrm{En}$ & 16 & -0.35 & $-1.05,0.35$ & $\mathrm{C}$ & Proficient & 4 & $\mathrm{~V}$ \\
\hline \multicolumn{9}{|l|}{ Vaid $(2001)^{\mathrm{d}}$} \\
\hline Group 1 & Sp-En & 10 & 0.08 & $-0.80,0.96$ & $\mathrm{C}$ & Proficient & 1 & DT \\
\hline Group 2 & Sp-En & 19 & 0.05 & $-0.59,0.68$ & $\mathrm{C}$ & Proficient & 1 & DT \\
\hline Vaid $(1999)^{d}$ & $\mathrm{Hi} / \mathrm{Ur}-\mathrm{En}$ & 10 & 00.61 & $-0.29,1.50$ & I & Proficient & 4 & $\mathrm{~V}$ \\
\hline Vaid $(1988)^{a}$ & Hi-En & 20 & 0.08 & $-0.54,0.70$ & $\mathrm{C}$ & Proficient & 4 & $\mathrm{~V}$ \\
\hline \multicolumn{9}{|l|}{ Vaid $(1987)^{\mathrm{a}}$} \\
\hline Group 1 & $\mathrm{En}-\mathrm{Fr}$ & 16 & -0.10 & $-0.78,0.59$ & I & Proficient & 4 & $\mathrm{~V}$ \\
\hline Group 2 & Fr-En & 16 & 0.18 & $-0.80,1.16$ & A & Proficient & 4 & $\mathrm{~V}$ \\
\hline \multicolumn{9}{|l|}{ Vaid (1984a; Exp. 1) } \\
\hline Group 1 & $\mathrm{En}-\mathrm{Fr}$ & 4 & -0.08 & $-1.46,1.31$ & A & Proficient & 3 & $\mathrm{~V}$ \\
\hline Group 2 & $\mathrm{En}-\mathrm{Fr}$ & 4 & -0.74 & $-2.17,0.70$ & A & Proficient & 3 & V \\
\hline Group 3 & Fr-En & 4 & -0.03 & $-1.42,1.35$ & A & Proficient & 3 & $\mathrm{~V}$ \\
\hline Group 4 & Fr-En & 4 & -0.11 & $-1.50,1.27$ & A & Proficient & 3 & V \\
\hline Group 5 & $\mathrm{En}-\mathrm{Fr}$ & 8 & -0.23 & $-1.21,0.76$ & I & Proficient & 3 & $\mathrm{~V}$ \\
\hline Group 6 & $\mathrm{En}-\mathrm{Fr}$ & 8 & -0.44 & $-1.43,0.56$ & I & Proficient & 3 & $\mathrm{~V}$ \\
\hline Vaid (1984a; Exp. 2) & $\mathrm{En}-\mathrm{Fr}$ & 8 & -0.36 & $-1.35,0.62$ & I & Proficient & 4 & $\mathrm{~V}$ \\
\hline \multicolumn{9}{|l|}{ Vaid (1984a; Exp. 3) } \\
\hline Group 1 & $\mathrm{En}-\mathrm{Fr}$ & 8 & -0.21 & $-1.19,0.78$ & I & Proficient & 4 & $\mathrm{~V}$ \\
\hline Group 2 & Fr-En & 8 & 0.11 & $-1.27,1.50$ & A & Proficient & 4 & $\mathrm{~V}$ \\
\hline \multicolumn{9}{|l|}{ Vaid $(1984 b)^{d}$} \\
\hline Group 1 & Fr-En & 8 & -0.43 & $-1.42,0.56$ & I & Proficient & 4 & V \\
\hline Group 2 & Fr-En & 8 & -0.17 & $-1.16,0.81$ & I & Proficient & 4 & $\mathrm{~V}$ \\
\hline \multicolumn{9}{|l|}{ Vaid (1981a) ${ }^{\mathrm{d}}$} \\
\hline Group 1 & $\mathrm{En}-\mathrm{Fr}$ & 8 & 0.17 & $-0.81,1.15$ & I & Proficient & 2 & $\mathrm{~V}$ \\
\hline Group 2 & Fr-En & 4 & 0.30 & $-1.10,1.69$ & A & Proficient & 2 & V \\
\hline Group 3 & $\mathrm{En}-\mathrm{Fr}$ & 4 & -0.02 & $-1.40,1.37$ & A & Proficient & 2 & $\mathrm{~V}$ \\
\hline \multicolumn{9}{|l|}{ Vaid $(1981 b)^{d}$} \\
\hline Group 1 & Fr-En & 8 & 0.06 & $-0.92,1.04$ & I & Proficient & 2 & $\mathrm{~V}$ \\
\hline Group 2 & Fr-En & 8 & 0.41 & $-0.58,1.40$ & A & Proficient & 2 & $\mathrm{~V}$ \\
\hline \multicolumn{9}{|l|}{ Vaid (1980a) ${ }^{\mathrm{d}}$} \\
\hline Group 1 & En-Fr & 8 & -0.24 & $-1.22,0.74$ & I & Proficient & 2 & V \\
\hline Group 2 & $\mathrm{En}-\mathrm{Fr}$ & 8 & -0.23 & $-1.21,0.76$ & I & Proficient & 2 & $\mathrm{~V}$ \\
\hline Group 3 & Fr-En & 4 & 0.15 & $-1.24,1.53$ & $\mathrm{~A}$ & Proficient & 2 & $\mathrm{~V}$ \\
\hline Group 4 & Fr-En & 4 & 0.12 & $-1.27,1.50$ & A & Proficient & 2 & $\mathrm{~V}$ \\
\hline Group 5 & $\mathrm{En}-\mathrm{Fr}$ & 4 & -0.01 & $-1.40,1.38$ & A & Proficient & 2 & $\mathrm{~V}$ \\
\hline Group 6 & $\mathrm{En}-\mathrm{Fr}$ & 4 & 0.29 & $-1.10,1.68$ & A & Proficient & 2 & $\mathrm{~V}$ \\
\hline \multicolumn{9}{|l|}{ Vaid $(1980 b)^{d}$} \\
\hline Group 1 & Fr-En & 8 & 0.69 & $-0.32,1.70$ & I & Proficient & 4 & V \\
\hline
\end{tabular}


Table 1 (Continued)

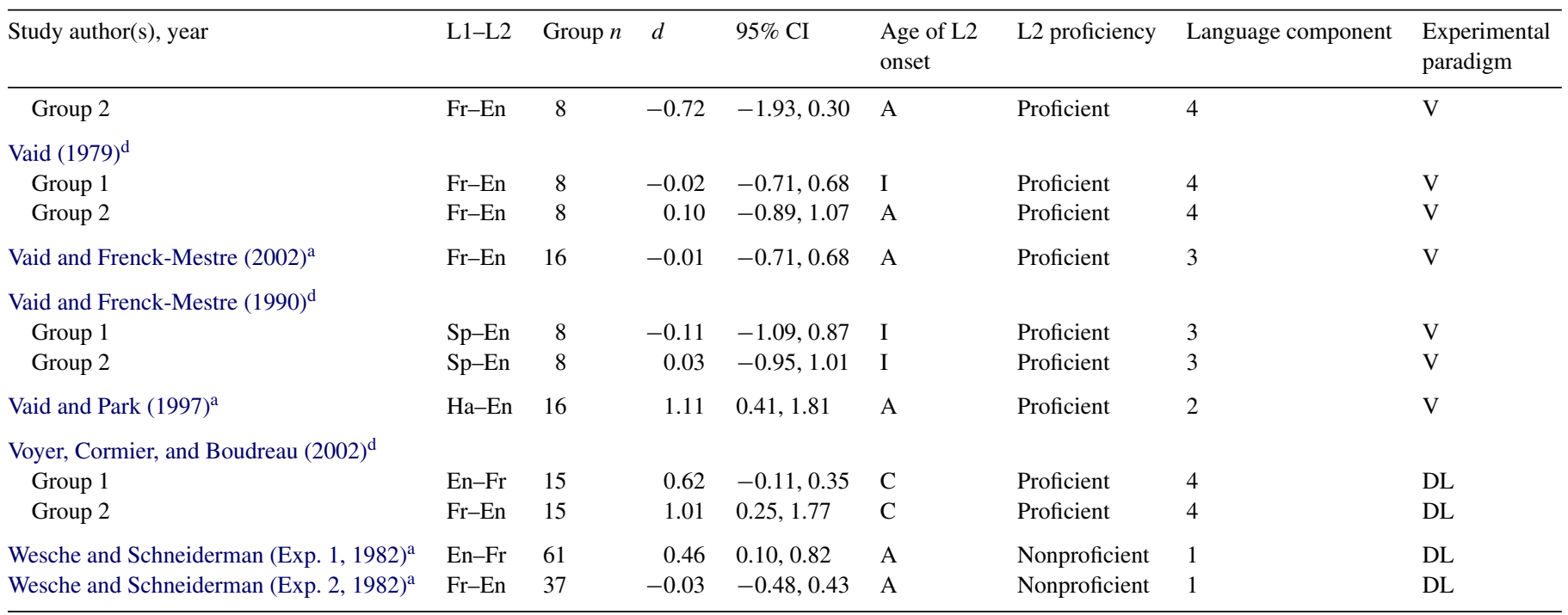

Note: CI: confidence interval; Exp.: experiment; DL: dichotic listening; DT: dual task; V: visual preference; I: infancy; C: childhood; A: adulthood. Effect sizes (ds) reflect greater activation in the left hemisphere when the CIs do not include zero, and bilateral activation when the CIs include zero. Language component key: (1) responses to single words; (2) phonetic judgments; (3) orthographic judgments; (4) semantic judgments; (5) syntactic judgments; (6) whole sentence comprehension. Language abbreviations key: En: English; Sp: Spanish; Fr: French; Ch: Chinese; Ma: Mandarin; Ge: German; Ru: Russian; Ca: Catalan; Ja: Japanese; It: Italian; Fi: Finnish; He: Hebrew; Kann: Kannada; Ka: Kanji; Ha: Harean; Fri: Friulan; Sw: Swedish; Po: Polish; Por: Portuguese; Vi: Vietnamese; Ur: Urdu; Hi: Hindi; Tu: Turkish; Ja: Japanese; Na: Navajo; To: Tok Pisin.

${ }^{a}$ Published study.

${ }^{\mathrm{b}}$ Unpublished thesis/dissertaion.

${ }^{\mathrm{c}}$ L2 acquisition age was not explicitly stated but was inferred from the text.

${ }^{\mathrm{d}}$ Unpublished study obtained from study author(s).

\subsection{Experimental approaches}

The present research included behavioral language laterality studies that used dichotic listening (DL), visual hemifield (V), or dual task (DT) paradigms to assess hemispheric involvement. The dichotic listening paradigm is a perceptionbased measure that involves the recall accuracy or reaction time (RT) of participants' auditory judgments of language stimuli simultaneously presented in either ear. Like dichotic listening, the visual hemifield tasks are based on participants' responses to unilaterally presented language stimuli, in this case to the right or left visual field. The dual task paradigm, also called the verbal-manual interference task, is presumed to reflect hemispheric involvement through decreased motor performance in finger-tapping rates for the hand that is associated with the hemisphere mediating the concurrent verbal task (i.e., the one that is contralateral to the hand). Thus, dual-task is a production-based paradigm.

Each of these methods relies on contralateral involvement to infer hemispheric participation, and there is evidence that each paradigm is suitable for inferring laterality of language function when properly used (see Hellige \& Sergent, 1986; Kosaka et al., 1993; Segalowitz, 1986). However, there is also reason to suspect systematic differences in hemispheric involvement related to the particular task or type of stimuli (see Boles, 2005) as well as the aspect of language engaged by a given paradigm (see Poeppel \& Hickok, 2004; Vaid, 1983). Consequently, the divisions of paradigm and language component (discussed in the following section) coded in the present research reflect a careful effort at precision in identifying potentially important methodological differences across studies that have not been systematically examined in this literature (see discussion in Boles, 2005).

\subsection{Language components tested}

The following six divisions of language were coded in each of the primary studies: (a) single word, global judgments, (b) word pair level phonological judgments, (c) word pair orthographic judgments, (d) word pair semantic judgments, (e) word pair syntactic judgments, and (f) sentence level judgments. It

is recognized that the divisions of language tested here represent only a subset of all possible divisions, and that it could be argued that categories such as "syntactic" or "semantic" themselves may need to be further fractionated (see Poeppel \& Hickok, 2004), but this research serves as an initial effort to consider the role of linguistic demands in laterality effects. Moreover, although other divisions are certainly possible (e.g., concreteness-abstractness, word frequency), these divisions have been infrequently examined in bilingual laterality studies.

\subsection{English as a second language}

Because English was the L2 in over half of the studies in the sample, it was possible to test whether language-specific laterality effects might differentially emerge for English relative to other L2s. That is, if language-specific factors matter for functional language organization, as suggested in the literature (e.g., Obler, 1981; Vaid \& Genesee, 1980), then the present English-heavy L2 sample could disproportionately influence the overall meta-analytic results in the direction of any effects specific to the English language. The variety of other L2s in the sample made it impractical to attempt similar examinations of each, as none represented a considerable percentage of the sample and would generally have resulted in very small cell sizes. Therefore, L2 data were coded and analyzed simply in terms of whether English or "other" was the L2.

The present study did not code or analyze for language-specific differences in L1, as we have previously reported a meta-analysis (Hull \& Vaid, 2006) that specifically investigated L1 laterality for a subset of the present data (i.e., 23 studies that included monolingual control groups). The previous meta-analysis showed that, whereas significant laterality differences emerged between infantonset bilinguals relative to monolinguals and late-onset bilinguals (who did not differ from each other), homogeneity was retained across L1s within each group, indicating no significant laterality differences despite differences among specific L1s. Therefore, in the present research, we sought to provide a substantive contribution to the literature by investigating an unanswered question, namely, whether learning English as a second language might matter to functional language lateralization. 
Table 2

Data for bilingual L2 laterality

\begin{tabular}{|c|c|c|c|c|c|c|c|c|}
\hline Study author(s), year & $\mathrm{L} 1-\mathrm{L} 2$ & Group $n$ & $d$ & $95 \% \mathrm{CI}$ & $\begin{array}{l}\text { Age of L2 } \\
\text { onset }\end{array}$ & L2 proficiency & $\begin{array}{l}\text { Language } \\
\text { component }\end{array}$ & $\begin{array}{l}\text { Experimental } \\
\text { paradigm }\end{array}$ \\
\hline \multicolumn{9}{|l|}{ Albanese $(1985)^{\mathrm{a}, \mathrm{c}}$} \\
\hline Group 1 & En-Fr & 10 & -0.22 & $-1.04,0.66$ & I & Proficient & 1 & DL \\
\hline Group 2 & En-Fr & 10 & 0.59 & $-0.30,1.49$ & A & Proficient & 1 & DL \\
\hline Bentin $(1981)^{\mathrm{a}, \mathrm{c}}$ & $\mathrm{He}-\mathrm{En}$ & 32 & 0.50 & $0.00,0.10$ & $\mathrm{C}$ & Nonproficient & 1 & $\mathrm{~V}$ \\
\hline Chengappa and Ray (2002) ${ }^{\mathrm{d}}$ & Kann-En & 10 & -0.38 & $-1.26,0.50$ & $\mathrm{C}$ & Proficient & 1 & $\mathrm{~V}$ \\
\hline Endo et al. $(1981 a)^{a}$ & $\mathrm{Ka}-\mathrm{Ha}$ & 13 & 0.47 & $-0.31,1.25$ & A & Nonproficient & 1 & DL \\
\hline Endo, Shimizu, and Nakamura (1981b) ${ }^{\mathrm{a}}$ & $\mathrm{Ja}-\mathrm{Ha}$ & 18 & 0.20 & $-0.45,0.86$ & A & Nonproficient & 1 & $\mathrm{~V}$ \\
\hline Fabbro (1992, Exp. 2) ${ }^{\mathrm{a}}$ & It-Ge & 3 & 1.00 & $-0.69,2.70$ & A & Proficient & 6 & DL \\
\hline \multicolumn{9}{|l|}{ Fabbro (1992, Exp. 4) ${ }^{\mathrm{a}}$} \\
\hline Group 1 & Fri-It & 12 & 0.17 & $-0.63,0.97$ & I & Proficient & 6 & DL \\
\hline Group 2 & Fri-It & 12 & 0.08 & $-0.72,0.88$ & I & Proficient & 6 & DL \\
\hline Group 3 & Fri-It & 12 & -0.03 & $-0.83,0.77$ & I & Proficient & 6 & DL \\
\hline Fabbro et al. $(1991)^{\mathrm{a}}$ & It-En & 36 & -0.36 & $-0.82,0.11$ & A & Proficient & 6 & DL \\
\hline Fabbro et al. (1990) ${ }^{\mathrm{a}}$ & It-En & 14 & 0.63 & $-0.13,1.40$ & A & Proficient & 6 & $\mathrm{DL}$ \\
\hline \multicolumn{9}{|l|}{ Fabbro et al. $(1988)^{\mathrm{a}}$} \\
\hline Group 1 & It-En & 12 & 0.81 & $-0.02,1.64$ & A & Proficient & 1 & DL \\
\hline Group 2 & It-En & 12 & 0.42 & $-0.39,1.23$ & A & Proficient & 1 & DL \\
\hline \multicolumn{9}{|l|}{ Furtado and Webster $(1991)^{\mathrm{a}}$} \\
\hline Group 1 & En-Fr & 16 & 0.06 & $-0.64,0.75$ & I & Proficient & 6 & DL \\
\hline Group 2 & En-Fr & 16 & 0.06 & $-0.64,0.75$ & A & Proficient & 6 & DL \\
\hline Group 3 & Fr-En & 16 & 0.00 & $-0.69,0.69$ & A & Proficient & 6 & DL \\
\hline \multicolumn{9}{|l|}{ Green $(1986)^{\mathrm{a}}$} \\
\hline Group 1 & En-Sp & 24 & 0.06 & $-0.51,0.62$ & A & Proficient & 6 & DL \\
\hline Group 2 & En-Sp & 24 & -0.25 & $-0.82,0.31$ & A & Nonproficient & 6 & DL \\
\hline Group 3 & En-Sp & 24 & 0.00 & $-0.57,0.57$ & A & Nonproficient & 6 & DL \\
\hline \multicolumn{9}{|l|}{ Green et al. $(1990)^{\mathrm{a}}$} \\
\hline Group 1 & $\mathrm{Sp}-\mathrm{En}$ & 8 & 0.40 & $-0.59,1.39$ & $\mathrm{C}$ & Proficient & 6 & DL \\
\hline Group 2 & Sp-En & 8 & 0.19 & $-0.79,1.18$ & $\mathrm{C}$ & Proficient & 6 & DL \\
\hline Group 3 & $\mathrm{Sp}-\mathrm{En}$ & 8 & 1.11 & $0.05,2.16$ & $\mathrm{C}$ & Proficient & 6 & DL \\
\hline Group 4 & Sp-En & 8 & 0.53 & $-0.47,1.53$ & $\mathrm{C}$ & Proficient & 6 & DL \\
\hline \multicolumn{9}{|l|}{ Hall and Lambert (1988) ${ }^{\mathrm{a}}$} \\
\hline Group 1 & En-Fr & 16 & 0.19 & $-0.50,0.89$ & $\mathrm{C}$ & Proficient & 6 & DL \\
\hline Group 2 & En-Fr & 16 & 0.22 & $-0.48,0.91$ & $\mathrm{C}$ & Proficient & 6 & DL \\
\hline Group 3 & $\mathrm{En}-\mathrm{Fr}$ & 16 & 0.24 & $-0.46,0.93$ & $\mathrm{C}$ & Nonproficient & 6 & DL \\
\hline Hatta $(1982)^{d}$ & $\mathrm{Ja}-\mathrm{En}$ & 20 & 0.55 & $-0.08,1.18$ & A & Nonproficient & 1 & DL \\
\hline $\begin{array}{l}\text { Hausmann, Durmusoglu, Yazgan, and } \\
\text { Güntürkün }(2004)^{\mathrm{a}}\end{array}$ & $\mathrm{Ge}-\mathrm{Tu}$ & 17 & 0.16 & $-0.52,0.83$ & I & Proficient & 4 & $\mathrm{~V}$ \\
\hline Hoosain and Shiu (1989) ${ }^{\mathrm{a}, \mathrm{c}}$ & $\mathrm{Ch}-\mathrm{En}$ & 28 & 0.38 & $-0.15,0.91$ & $\mathrm{C}$ & Proficient & 1 & $\mathrm{~V}$ \\
\hline \multicolumn{9}{|l|}{ Ip and Hoosain $(1993)^{\mathrm{a}, \mathrm{c}}$} \\
\hline Group 1 & $\mathrm{Ch}-\mathrm{En}$ & 9 & 0.41 & $-0.52,1.34$ & $\mathrm{C}$ & Proficient & 1 & DL \\
\hline Group 2 & Ch-En & 9 & 0.26 & $-0.67,1.18$ & $\mathrm{C}$ & Proficient & 1 & DL \\
\hline \multicolumn{9}{|l|}{$\operatorname{Jin}(1988)^{\mathrm{b}, \mathrm{c}}$} \\
\hline Group 1 & $\mathrm{Ha}-\mathrm{En}-\mathrm{Ch}$ & 24 & 0.79 & $0.21,1.38$ & A & Proficient & 3 & $\mathrm{~V}$ \\
\hline Group 2 & $\mathrm{Ha}-\mathrm{En}-\mathrm{Ch}$ & 24 & -0.67 & $-1.25,-.09$ & A & Nonproficient & 3 & $\mathrm{~V}$ \\
\hline Kang $(1984)^{b}$ & Misc.-En & 40 & 0.68 & $0.23,1.13$ & A & Proficient & 1 & DL \\
\hline $\operatorname{Ke}(1992)^{\mathrm{a}, \mathrm{c}}$ & $\mathrm{En}-\mathrm{Ch}$ & 28 & 0.31 & $-0.22,0.84$ & A & Proficient & 1 & DL \\
\hline \multicolumn{9}{|l|}{ Kilborn $(2002)^{\mathrm{d}}$} \\
\hline Group 1 & $\mathrm{Ur}-\mathrm{En}$ & 60 & 0.02 & $-0.34,0.38$ & I & Proficient & 1 & $\mathrm{~V}$ \\
\hline Group 2 & $\mathrm{Ur}-\mathrm{En}$ & 60 & -0.05 & $-0.41,0.31$ & A & Proficient & 1 & $\mathrm{~V}$ \\
\hline \multicolumn{9}{|l|}{ Mägiste (1989)a } \\
\hline Group 1 & & 9 & -0.29 & $-1.21,0.64$ & $\mathrm{C}$ & Proficient & 6 & $\mathrm{~V}$ \\
\hline Group 2 & & 9 & -0.16 & $-1.08,0.77$ & $\mathrm{C}$ & Proficient & 6 & $\mathrm{~V}$ \\
\hline \multicolumn{9}{|l|}{ Mägiste $(1987)^{\mathrm{a}, \mathrm{c}}$} \\
\hline Group 1 & $\mathrm{Ge}-\mathrm{Sw}$ & 10 & 0.41 & $-0.48,1.29$ & I & Proficient & 1 & $\mathrm{~V}$ \\
\hline Group 2 & $\mathrm{Ge}-\mathrm{Sw}$ & 10 & 0.35 & $-0.53,1.23$ & A & Proficient & 1 & $\mathrm{~V}$ \\
\hline
\end{tabular}


Table 2 (Continued)

\begin{tabular}{|c|c|c|c|c|c|c|c|c|}
\hline Study author(s), year & L1-L2 & Group $n$ & $d$ & $95 \% \mathrm{CI}$ & $\begin{array}{l}\text { Age of L2 } \\
\text { onset }\end{array}$ & L2 proficiency & $\begin{array}{l}\text { Language } \\
\text { component }\end{array}$ & $\begin{array}{l}\text { Experimental } \\
\text { paradigm }\end{array}$ \\
\hline Group 3 & $\mathrm{Po}-\mathrm{Sw}$ & 14 & -0.07 & $-0.81,0.67$ & A & Nonproficient & 1 & $\mathrm{~V}$ \\
\hline Manga and Sanchez (1989) $)^{\mathrm{a}, \mathrm{c}}$ & En-Sp & 31 & 0.38 & $-0.12,0.87$ & $\mathrm{C}$ & Proficient & 6 & DL \\
\hline \multicolumn{9}{|c|}{ Persinger, Chellew-Belanger, and Tiller (2002) ${ }^{\mathrm{a}}$} \\
\hline Group 1 & Fr-En & 15 & 1.42 & $0.62,2.22$ & A & Proficient & 1 & DL \\
\hline Group 2 & Fr-En & 18 & 0.32 & $-0.34,0.98$ & A & Proficient & 1 & DL \\
\hline Rastatter and Scukanec $(1990)^{\mathrm{a}}$ & $\mathrm{Ch}-\mathrm{En}$ & 16 & 0.35 & $-0.35,1.05$ & $\mathrm{C}$ & Proficient & 1 & $\mathrm{~V}$ \\
\hline $\operatorname{Rupp}(1980)^{\mathrm{b}}$ & Vi-En & 86 & 0.92 & $0.61,1.24$ & $\mathrm{C}$ & Nonproficient & 1 & DL \\
\hline \multicolumn{9}{|l|}{ Sewell and Panou (1983) ${ }^{\mathrm{a}}$} \\
\hline Group 1 & En-Ge & 6 & 0.22 & $-0.91,1.36$ & A & Proficient & 1 & $\mathrm{~V}$ \\
\hline Group 2 & $\mathrm{En}-\mathrm{Ge}$ & 6 & 0.45 & $-0.70,1.59$ & A & Proficient & 1 & $\mathrm{~V}$ \\
\hline Group 3 & $\mathrm{En}-\mathrm{Fr}$ & 6 & 0.14 & $-0.99,1.28$ & I & Proficient & 1 & $\mathrm{~V}$ \\
\hline Group 4 & $\mathrm{En}-\mathrm{Fr}$ & 6 & 0.21 & $-0.93,1.34$ & I & Proficient & 1 & $\mathrm{~V}$ \\
\hline \multicolumn{9}{|l|}{ Shanon $(1982)^{\mathrm{a}}$} \\
\hline Group 1a & $\mathrm{He}-\mathrm{En}$ & 8 & -0.17 & $-1.15,0.81$ & A & Proficient & 1 & $\mathrm{~V}$ \\
\hline Group $1 b$ & $\mathrm{He}-\mathrm{En}$ & 8 & -0.23 & $-1.22,0.75$ & A & Proficient & 1 & $\mathrm{~V}$ \\
\hline Group 2a & $\mathrm{He}-\mathrm{En}$ & 8 & 0.70 & $-0.31,1.71$ & I & Proficient & 1 & $\mathrm{~V}$ \\
\hline Group $2 b$ & $\mathrm{He}-\mathrm{En}$ & 8 & 0.35 & $-0.64,1.33$ & I & Proficient & 1 & $\mathrm{~V}$ \\
\hline Group 3a & $\mathrm{En}-\mathrm{He}$ & 8 & 0.22 & $0.76,1.20$ & A & Proficient & 1 & $\mathrm{~V}$ \\
\hline Group $3 b$ & $\mathrm{En}-\mathrm{He}$ & 8 & -0.31 & $-1.30,0.67$ & A & Proficient & 1 & $\mathrm{~V}$ \\
\hline Soares $(1984)^{\mathrm{a}}$ & Por-En & 16 & 0.93 & $0.20,1.66$ & A & Proficient & 6 & DT \\
\hline \multicolumn{9}{|l|}{ Spiller-Bosatra et al. $(1990)^{\mathrm{a}}$} \\
\hline Group 1 & $\mathrm{It}-\mathrm{Ge}$ & 3 & -0.47 & $-2.09,1.15$ & I & Proficient & 1 & DL \\
\hline Group 2 & It-Ge & 5 & -0.02 & $-1.26,1.22$ & I & Proficient & 1 & DL \\
\hline Thomas $(1987)^{b, c}$ & $\mathrm{Ch}-\mathrm{En}$ & 26 & 0.20 & $-0.34,0.75$ & A & Proficient & 1 & DL \\
\hline \multicolumn{9}{|l|}{ Vaid $(2003)^{\mathrm{d}}$} \\
\hline Group 1 & $\mathrm{Hi}-\mathrm{En}$ & 16 & -0.14 & $-0.84,0.55$ & $\mathrm{C}$ & Proficient & 4 & $\mathrm{~V}$ \\
\hline Group 2 & Ur-En & 16 & -0.08 & $-0.77,0.61$ & $\mathrm{C}$ & Proficient & 4 & $\mathrm{~V}$ \\
\hline \multicolumn{9}{|l|}{ Vaid $(2001)^{\mathrm{d}}$} \\
\hline Group 1 & Sp-En & 10 & 0.20 & $-0.68,1.07$ & $\mathrm{C}$ & Proficient & 1 & DL \\
\hline Group 2 & Sp-En & 19 & 0.05 & $-0.59,0.69$ & $\mathrm{C}$ & Proficient & 1 & DL \\
\hline Vaid (1999) ${ }^{\mathrm{d}}$ & $\mathrm{Hi}-\mathrm{En}$ & 10 & 0.41 & $-0.47,1.30$ & I & Proficient & 4 & $\mathrm{~V}$ \\
\hline \multicolumn{9}{|l|}{ Vaid (1984a; Exp. 1) } \\
\hline Group 1 & En-Fr & 4 & -0.03 & $-1.41,1.36$ & A & Proficient & 3 & $\mathrm{~V}$ \\
\hline Group 2 & $\mathrm{En}-\mathrm{Fr}$ & 4 & 0.09 & $-1.30,1.47$ & A & Proficient & 3 & $\mathrm{~V}$ \\
\hline Group 3 & Fr-En & 4 & 0.06 & $-1.33,1.45$ & A & Proficient & 3 & $\mathrm{~V}$ \\
\hline Group 4 & Fr-En & 4 & -0.30 & $-1.69,1.10$ & A & Proficient & 3 & $\mathrm{~V}$ \\
\hline Group 5 & $\mathrm{En}-\mathrm{Fr}$ & 8 & -0.43 & $-1.42,0.57$ & I & Proficient & 3 & $\mathrm{~V}$ \\
\hline Group 6 & $\mathrm{En}-\mathrm{Fr}$ & 8 & -0.10 & $-1.08,0.89$ & I & Proficient & 3 & $\mathrm{~V}$ \\
\hline \multicolumn{9}{|l|}{ Vaid (1984a; Exp. 3) } \\
\hline Group 1 & $\mathrm{En}-\mathrm{Fr}$ & 8 & -0.21 & $-1.19,0.78$ & I & Proficient & 4 & $\mathrm{~V}$ \\
\hline Group 2 & $\mathrm{En}-\mathrm{Fr}$ & 4 & 0.27 & $-1.12,1.66$ & A & Proficient & 4 & $\mathrm{~V}$ \\
\hline Group 3 & Fr-En & 4 & -0.01 & $-1.39,1.38$ & A & Proficient & 4 & $\mathrm{~V}$ \\
\hline \multicolumn{9}{|l|}{ Vaid (1981a) } \\
\hline Group 1 & $\mathrm{En}-\mathrm{Fr}$ & 8 & 0.20 & $-0.78,1.19$ & I & Proficient & 2 & $\mathrm{~V}$ \\
\hline Group 2 & Fr-En & 4 & -0.17 & $-1.56,1.22$ & A & Proficient & 2 & $\mathrm{~V}$ \\
\hline Group 3 & En-Fr & 4 & 0.20 & $-1.19,1.59$ & A & Proficient & 2 & $\mathrm{~V}$ \\
\hline \multicolumn{9}{|l|}{ Vaid (1981b) ${ }^{\mathrm{d}}$} \\
\hline Group 1 & Fr-En & 8 & 0.45 & $-0.55,1.44$ & I & Proficient & 2 & $\mathrm{~V}$ \\
\hline Group 2 & Fr-En & 8 & 0.68 & $-0.33,1.69$ & A & Proficient & 2 & $\mathrm{~V}$ \\
\hline \multicolumn{9}{|l|}{ Vaid $(1980 a)^{d}$} \\
\hline Group 1 & $\mathrm{En}-\mathrm{Fr}$ & 8 & -0.24 & $-1.22,0.74$ & I & Proficient & 2 & $\mathrm{~V}$ \\
\hline Group 2 & $\mathrm{En}-\mathrm{Fr}$ & 8 & -0.23 & $-1.21,0.76$ & I & Proficient & 2 & $\mathrm{~V}$ \\
\hline Group 3 & Fr-En & 4 & 0.34 & $-1.06,1.74$ & A & Proficient & 2 & $\mathrm{~V}$ \\
\hline Group 4 & Fr-En & 4 & 0.14 & $-1.25,1.52$ & A & Proficient & 2 & $\mathrm{~V}$ \\
\hline Group 5 & $\mathrm{En}-\mathrm{Fr}$ & 4 & -0.12 & $-1.51,1.27$ & A & Proficient & 2 & $\mathrm{~V}$ \\
\hline Group 6 & $\mathrm{En}-\mathrm{Fr}$ & 4 & -0.02 & $-1.40,1.37$ & A & Proficient & 2 & $\mathrm{~V}$ \\
\hline
\end{tabular}


Table 2 (Continued)

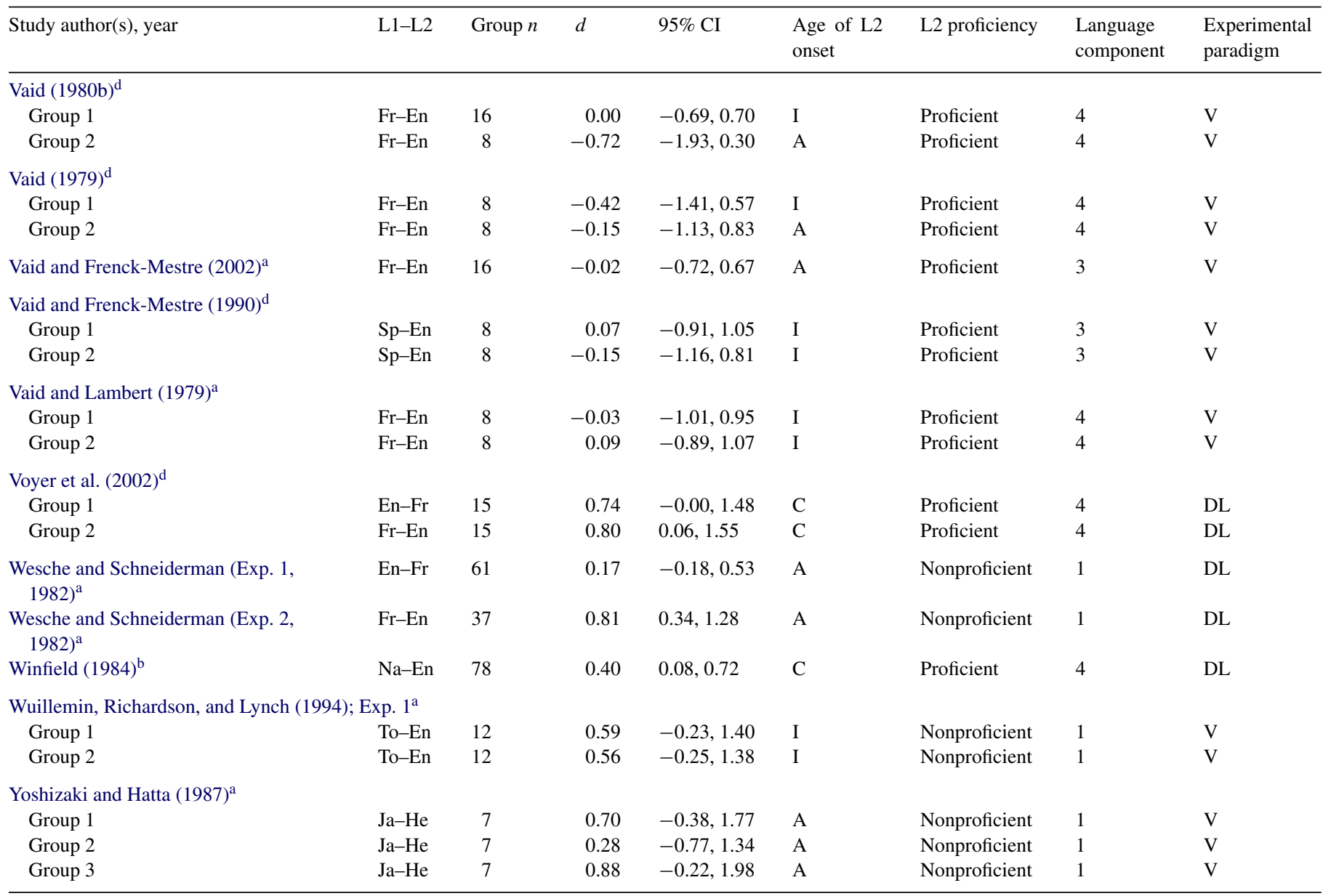

Note: CI: confidence interval; Exp.: experiment; DL: dichotic listening; DT: dual task; V: visual preference; I: infancy; C: childhood; A: adulthood. Effect sizes ( $d$ s) reflect greater activation in the left hemisphere when the CIs do not include zero, and bilateral activation when the CIs include zero. Language component key: (1) responses to single words; (2) phonetic judgments; (3) orthographic judgments; (4) semantic judgments; (5) syntactic judgments; (6) whole sentence comprehension. Language abbreviations key: En: English; Sp: Spanish; Fr: French; Ch: Chinese; Ma: Mandarin; Ge: German; Ru: Russian; Ca: Catalan; Ja: Japanese; It: Italian; Fi: Finnish; He: Hebrew; Kann: Kannada; Ka: Kanji; Ha: Harean; Fri: Friulan; Sw: Swedish; Po: Polish; Por: Portuguese; Vi: Vietnamese; Ur: Urdu; Hi: Hindi; Tu: Turkish; Ja: Japanese; Na: Navajo; To: Tok Pisin.

a Published study.

b Unpublished thesis/dissertation.

c L2 acquisition age was not explicitly stated but was inferred from the text.

d Unpublished study obtained from study author(s).

\subsection{Calculation of effect sizes}

The meta-analyses standardize scores from different outcome measures (e.g., accuracy versus reaction time) into effect size statistics so that meaningful numerical comparisons and analyses of means and variance can be made across studies (Lipsey \& Wilson, 2001). All included studies were characterized by direct comparisons of two or more groups, rather than by correlational designs. Accordingly, the effect size statistic used to measure the strength of the independent variables in the present research was Cohen's $d$ (Cohen, 1992). Following Hedges and Olkin (1985), effect sizes were calculated by taking the difference between the control and experimental means and dividing by the pooled standard deviation, shown algebraically as $\left(M_{1}-M_{2}\right) / \delta$. Specifically, group data associated with LH performance (e.g., mean tapping rate with the right hand, listening accuracy with the right ear, or reporting accuracy from the right visual field) were treated as the control condition $\left(M_{1}\right)$, and data associated with the RH were treated as the experimental condition $\left(M_{2}\right)$. A positive effect size is associated with greater LH involvement, a negative effect size with greater RH involvement, and effect sizes near zero with bilateral symmetry. The effect sizes

were adjusted for bias in population effect size by converting the raw $g \mathrm{~s}$ to $d \mathrm{~s}$ (Hedges and Olkin).

Once computed, the effect sizes were weighted by sample size and aggregated into the appropriate divisions of each moderating variable (e.g., one mean effect size represented all bilinguals with late $\mathrm{L} 2$ acquisition). The resulting aggregated $d$ thus provided a summary of the magnitude and direction of each moderator's effects on functional language laterality. The weighting procedure produced a metric of sampling error that involved multiplying the raw effect size by the reciprocal of its variance. As a result, the more reliably estimated effect sizes (i.e., ones with relatively less variance) had more weight in the aggregate analysis. The associated $95 \%$ confidence intervals (CIs) were calculated to describe the range within which a given effect size was expected to fall $95 \%$ of the time (see Hedges \& Olkin, 1985).

The third step of the data analysis was to calculate the homogeneity statistic $Q$ for each division of each moderator. The $Q$, or "goodness-of-fit" statistic, represents an approximate chi-square distribution with degrees of freedom $k-1$, where $k$ is the total number of effect sizes in the set. $Q$ indicates whether the sample of $d$ s is similar enough to be considered one population. It should be 
noted that the power of $Q$ is compromised when set sizes are very small (e.g. Arthur et al., 2001; Lipsey \& Wilson, 2001) In the present research, effect sizes were considered homogenous only in cases where $Q$ indicated homogeneity and where at least six data points comprised the cell. Categorical modeling of the a priori moderator divisions was conducted when unexplained variance remained (Hunter \& Schmidt, 1990) and/or to address theoretical positions in the literature (Wood \& Quinn, 2003).

The categorical models supplied two types of $Q$ statistics; one tested homogeneity between moderator divisions, $Q_{\mathrm{B}}$, and the other within each division, $Q_{\mathrm{W}}$. In a categorical model, a significant $Q_{\mathrm{B}}$ statistic indicates that at least two moderator divisions derive from distinct populations. In such cases, direct contrasts (using the chi-square distribution with $k-1$ degrees of freedom) were conducted to identify significant differences among divisions (see Lipsey $\&$ Wilson, 2001). For example, if the $Q_{\mathrm{B}}$ statistic indicated heterogeneity within the model for experimental paradigm, then direct contrasts were conducted on the aggregate $d$ s for the three moderator divisions (visual preference, dichotic listening, and dual task) to determine which was different from which.

In cases where homogeneity could not be retained in a categorical model for a single moderating variable, it was assumed that at least one additional moderating variable was in operation, and further partitioning was performed in an attempt to account for the unexplained variance. That is, categorical models with all combinations of two moderators (e.g., language experience and paradigm) were calculated, then three moderators, and so forth, until homogeneity was retained, moderator categories were exhausted, or cell sizes became too small to yield reliable results.

\subsection{Results and discussion of moderating effects on L1 laterality}

A stem and leaf display is provided for mean effect sizes from the L1 meta-analysis (Fig. 1). The fixed-effects estimate of L1 laterality across 98 independent data points indicated a small LH advantage for L1s overall $(d=0.23$, $95 \% \mathrm{CI}=0.15,0.30 ; k=98)$, and the homogeneity statistic was marginal, $Q_{\mathrm{W}}(97)=121.45, p<0.09$. Categorical modeling for the coded moderators was undertaken to test for circumstances under which language laterality effects might have varied. A summary of the categorical models that best predicted L1 effect sizes is provided in Table 3 .

\subsubsection{Second language proficiency}

The effect of $\mathrm{L} 2$ proficiency revealed a very small but reliable LH laterality for proficient bilinguals overall $(d=0.15 ; 95 \% \mathrm{CI}=0.07,0.23 ; k=92)$, whereas nonproficient bilinguals were strongly and reliably LH lateralized $(d=0.68 ; 95 \%$ $\mathrm{CI}=0.48,0.88 ; k=6$ ). Indeed, nonproficient bilinguals were more $\mathrm{LH}$ dominant than proficient bilinguals, $Q_{\mathrm{B}}(1)=22.95, p<0.01$, a finding at odds with theoretical predictions for greater $\mathrm{LH}$ involvement with increased L2 proficiency (Galloway \& Krashen, 1980; Obler, 1981; Ullman, 2001). However, the strength of the LH effect for proficient bilinguals was undoubtedly weakened by the inclusion of infant onset bilinguals, for whom bilateral activation for language has previously been demonstrated (Hull \& Vaid, 2005; Hull \& Vaid, 2006) and for whom brain structural differences have been detected relative to late bilinguals (Mechelli et al., 2004). Therefore, the set of proficient bilinguals was partitioned by ages of bilingualism onset to test these theoretical predictions and empirical outcomes in the literature. The categorical model confirmed that proficient infant onset bilinguals were bilaterally activated for language $(d=-0.01 ; 95 \%$ $\mathrm{CI}=-0.17,0.14 ; k=31)$, whereas proficient adult onset bilinguals $(d=0.26$; $95 \% \mathrm{CI}=0.13,0.38 ; k=39)$ showed an LH effect that was significantly different from the infant onset group, $Q_{\mathrm{B}}(1)=7.13, p<0.01$. Interestingly, while proficient childhood onset bilinguals showed marginal bilateral activation for language $(d=0.14 ; 95 \% \mathrm{CI}=-0.02,0.30 ; k=22)$, they were not significantly different from the proficient adult onset group, $Q_{\mathrm{B}}(1)=1.38$, n.s., or the infant onset group $Q_{\mathrm{B}}(1)=1.75$, n.s. $<0.01$, suggesting that this middle ground on the temporal continuum of L2 acquisition may be reflected in an analogous middle ground in terms of functional language laterality.

\subsubsection{Age of bilingualism onset}

The effect of bilingualism onset age revealed LH lateralization for language in childhood $(d=0.31 ; 95 \% \mathrm{CI}=0.18,0.44 ; k=26)$ and adult onset bilinguals $(d=0.30 ; 95 \% \mathrm{CI}=0.17,0.41 ; k=41)$, whereas infant onset bilinguals were bilaterally activated $(d=-0.01 ; 95 \% \mathrm{CI}=-0.16,0.14 ; k=31)$. There was no difference in functional language laterality between the child and adult bilingual groups, $Q_{\mathrm{B}}(1)=0.03, p=0.86$, but both were significantly more LH lateralized than the infant group, $Q_{\mathrm{B}}(1)=9.79, p<0.01$, and $Q_{\mathrm{B}}(1)=9.47, p<0.01$, respectively. However, although homogeneity was retained within the adult onset group, $Q_{\mathrm{B}}(1)=18.76, p<0.01$, unexplained variance was present for the infant onset, $Q_{\mathrm{W}}(30)=12.72, p<0.01$, and child onset bilingual sub-samples, $Q_{\mathrm{W}}(25)=44.57, p<0.05$.

\subsubsection{English as the L2}

Given that models of $\mathrm{L} 2$ proficiency were moderated by age of bilingualism onset and that variance remained in infant and childhood bilingualism onset groups, further partitioning of L1 effect size variance in these groups was undertaken according to whether English was the L2. Results showed that homogeneity was retained within infant onset bilinguals when they were separately analyzed according to whether English was the $\mathrm{L} 2, Q_{\mathrm{W}}(11)=6.94$, $p=0.86$, or "other" was the $\mathrm{L} 2, Q_{\mathrm{W}}(18)=5.29, p=0.99$. Likewise, homogeneity was retained within the childhood subgroups when the L2 was English, $Q_{\mathrm{W}}(17)=25.50, p=0.11$, or "other," $Q_{\mathrm{W}}(7)=10.09, p=0.26$. Interestingly, when English was the L2, the L1s of childhood onset bilinguals were significantly more LH lateralized than the L1s of childhood bilinguals who had L2s other than English, $Q_{\mathrm{B}}(1)=8.99, p<0.01$. This pattern did not hold for infant onset, $Q_{\mathrm{B}}(1)=0.49, p=0.48$, or adult onset bilinguals, $Q_{\mathrm{B}}(1)=1.04, p=0.31$.

The above outcomes suggest a critical consideration for studies of functional language organization in the brain. Specifically, it appears that there may something particular about learning English as a second language that promotes more LH involvement relative to learning other languages (see Obler, 1981). One possible explanation for this finding may be sought in the irregularity of grapheme-to-phoneme correspondence in English relative to other Indo-European languages (Seymour, Aro, \& Erskine, 2003). That is, it may be that this feature of English orthography could make the reading of English more dependent on left-hemisphere mediated systems. Evidence from ERP and fMRI studies has suggested that irregular English words are processed differently from regular English words, with the latter relying more on bilateral temporal regions, and the former relying more on left frontal regions (Newman, Izvorski, Davis, Neville, \& Ullman, 1999; Ullman, Bergida, \& O'Craven, 1997). Other imaging evidence that may be considered consistent with our suggestion for Englishspecific effects can be found in a recent report of common activation for Finnish (L1) and English (L2) in precuneus, dorsolateral prefrontal cortex, Broca's area, angular/supramarginal gyri, and cerebellum in late bilinguals, but with generally increased LH activation during English use (Halsband, 2006). Halsband suggested this effect was a likely result of the markedly different linguistic structure of English (the L2) as compared to Finnish (the L1).

Whereas language-specific effects have generally been understudied in bilingual laterality research and thus could not be tested here, it is hoped that future laterality research will focus on finer parsing of the factors that influence laterality in childhood onset bilingualism. Nonetheless, it remains important that bilingualism onset age, taken together with the linguistic nature of the L2, explains all variance in the variety of behavioral laterality outcomes as reported in the literature thus far.

\subsubsection{Language component}

For L1s, 52\% of studies tested components of language thought to be preferentially processed in the RH (i.e., orthographic and semantic word pair judgments), while $48 \%$ focused on the remaining components (i.e., single word, phonological, syntactic, and sentence processing), which have been considered within the domain of the LH. Results revealed that orthographic $(d=0.18$; $\left.95 \% \mathrm{CI}=0.06,0.32 ; k=43, Q_{\mathrm{W}}(42)=40.36, p=0.81\right)$ and semantic processing $\left(d=0.13 ; 95 \% \mathrm{CI}=-0.09,0.35 ; k=12, Q_{\mathrm{W}}(11)=16.11, p=0.17\right)$ showed very weak LH lateralization and bilateral activation, respectively, consistent with predictions in the literature (e.g., Vaid, 1984a). In contrast, responses to single words $\left(d=0.34 ; 95 \% \mathrm{CI}=0.22,0.46 ; k=28, Q_{\mathrm{W}}(27)=46.85, p<0.05\right)$ showed increased LH involvement, also as predicted in the literature (e.g., Chiarello, Liu, Shears, \& Kacinik, 2002). For phonological word pair judgments, a reliable bilateral effect was detected $(d=0.32 ; 95 \% \mathrm{CI}=-0.06,0.70$; $\left.k=8, Q_{\mathrm{W}}(7)=7.10, p=0.53\right)$, consistent with some current theories of speech perception (e.g., Hickok \& Poeppel, 2004). Outcomes from syntactic word pair 


\begin{tabular}{|c|c|c|}
\hline Attachment & Stem & Leaf \\
\hline 3 & -.7 & 223 \\
\hline 0 & -.6 & 0 \\
\hline 1 & -.5 & 3 \\
\hline 2 & -.4 & 23 \\
\hline 3 & -.3 & 466 \\
\hline 9 & -.2 & 012234668 \\
\hline 8 & -.1 & 00111247 \\
\hline 11 & -.0 & 01111233778 \\
\hline 9 & .0 & 345678888 \\
\hline 15 & .1 & 112244456788999 \\
\hline 8 & .5 & 00168899 \\
\hline 4 & .3 & 0177 \\
\hline 8 & .4 & 04556789 \\
\hline 2 & .5 & 56 \\
\hline 5 & .6 & 0189 \\
\hline 2 & .7 & 57 \\
\hline 3 & .8 & 226 \\
\hline 30 & .9 & 0 \\
\hline 3 & 1. & 089 \\
\hline 2 & Extremes $>1.1$ & \\
\hline
\end{tabular}

Fig. 1. Stem and leaf display of effect sizes for L1 laterality.

judgments and sentence comprehension were both based on fewer than five data points and are not discussed further.

Homogeneity was not retained within outcomes from responses to single words (28 data points), thus supporting the notion that some discrepancies among bilingual laterality studies could arise from variations in the components of language tested. However, homogeneity was retained across divisions, $Q_{\mathrm{B}}(5)=10.60$, n.s., indicating that component of language, at least as could be defined in the present research, was probably not a significant source of overall variance in aggregated L1 effect sizes.

\subsubsection{Experimental paradigm}

Partitioning L1 variance by experimental paradigm revealed differential influences on bilingual laterality. Specifically, aggregated $d$ s within the visual preference paradigm showed a very small but reliable LH effect $(d=0.17 ; 95 \%$ $\mathrm{CI}=0.05,0.29 ; k=56), Q_{\mathrm{W}}(55)=47.68, p=0.44$, which did not differ from the reliable bilateral effect for the dual task paradigm $(d=0.12 ; 95 \% \mathrm{CI}=-0.02$, $0.26 ; k=25), Q_{\mathrm{W}}(24)=26.49, p=0.38$, as evidenced by the nonsignificant between-groups homogeneity statistic, $Q_{\mathrm{B}}(1)=0.23, p=0.63$. Notably, both of these paradigms were associated with significantly less $\mathrm{LH}$ lateralization than the dichotic listening paradigm, $Q_{\mathrm{B}}(1)=6.26, p<0.05$, and $Q_{\mathrm{B}}(1)=7.95, p<0.05$, respectively. Moreover, homogeneity was not retained within the dichotic listening paradigm $(d=0.41 ; 95 \% \mathrm{CI}=0.27,0.55 ; k=17), Q_{\mathrm{W}}(16)=37.96, p<0.01$. These outcomes replicate those from a previous meta-analysis of the effects of paradigm on language laterality (Hull \& Vaid, 2006). Given the present finding that single word processing was among the most strongly LH lateralized language components and that dichotic listening tasks typically involve single word processing, the finding that the dichotic listening paradigm induced a stronger $\mathrm{LH}$ effect on $\mathrm{L} 1$ processing relative to other paradigms is not surprising. Rather, it highlights the importance of investigating substantive reasons why laterality differences among paradigms might emerge.

\subsection{Results and discussion of moderating effects on L2 laterality}

A stem and leaf display is provided for mean effect sizes from the L2 metaanalysis (Fig. 2). The overall estimated effect size $(d=0.25 ; 95 \% \mathrm{CI}=0.18,0.32)$ from 106 independent data points indicated a small $\mathrm{LH}$ effect for $\mathrm{L} 2$ overall, and homogeneity was retained, $Q_{\mathrm{W}}(105)=123.92$, n.s. To address questions in the literature concerning the influence of language experience on language lateralization, categorical modeling of the coded moderators was carried out. A summary of the models that best predicted L2 laterality is provided in Table 4. 
Table 3

Mean effect size estimates for moderators of L1 laterality

\begin{tabular}{|c|c|c|c|c|c|c|}
\hline Moderator & $k$ & Total sample size $n$ & Sample weighted mean $d$ & $95 \% \mathrm{CI}$ & Mean unweighted $d$ & Homogeneity statistic $Q_{\mathrm{W}}$ \\
\hline Overall dataset & 98 & 1348 & 0.23 & $0.15,0.30$ & 0.16 & 121.45 \\
\hline \multicolumn{7}{|c|}{$\mathrm{L} 1$ dataset partitioned by age of $\mathrm{L} 2$ acquisition onset } \\
\hline Childhood & 26 & 470 & 0.31 & $0.18,0.44$ & 0.22 & $44.57^{*}$ \\
\hline Adulthood & 41 & 555 & 0.30 & $0.18,0.41$ & 0.26 & 52.17 \\
\hline \multicolumn{7}{|c|}{ Infant dataset partitioned by English as the L2 } \\
\hline \multicolumn{7}{|c|}{ Childhood dataset partitioned by English as the L2 } \\
\hline L2 English & 18 & & 0.43 & $0.28,0.58$ & & 25.50 \\
\hline L2 other & 8 & & -0.02 & $-0.28,0.23$ & & 10.09 \\
\hline \multicolumn{7}{|c|}{ L1 dataset partitioned by L2 proficiency } \\
\hline Proficient & 92 & 1146 & 0.15 & $0.07,0.23$ & 0.13 & 95.66 \\
\hline Dual task & 25 & 410 & 0.12 & $-0.02,0.26$ & 0.17 & 26.49 \\
\hline \multicolumn{7}{|c|}{ L1 dataset partitioned by linguistic task demands } \\
\hline Single words & 28 & 544 & 0.34 & $0.22,0.46$ & 0.26 & $46.85^{*}$ \\
\hline Phonological-word pairs & 8 & 56 & 0.32 & $-0.06,0.70$ & 0.20 & 7.10 \\
\hline Orthographic-word pairs & 43 & 482 & 0.19 & $0.06,0.32$ & 0.14 & 40.36 \\
\hline Semantic-word pairs & 12 & 164 & 0.13 & $-0.09,0.35$ & 0.11 & 16.11 \\
\hline
\end{tabular}

Note: ${ }^{*} p<0.05$, d.f. $=k-1 ; k=$ number of independent effect sizes; CI: confidence interval; L2: second language. Positive effect sizes ( $d$ s) reflect greater activation in the left hemisphere when the CIs do not include zero and bilateral activation when the CIs include zero.

Table 4

Mean effect size estimates for moderators of L2 laterality

\begin{tabular}{|c|c|c|c|c|c|c|}
\hline Moderator & $k$ & Total sample size $n$ & Sample weighted mean $d$ & $95 \% \mathrm{CI}$ & Mean unweighted $d$ & Homogeneity statistic $Q_{\mathrm{W}}$ \\
\hline Overall dataset & 106 & 1587 & 0.25 & $0.18,0.32$ & 0.20 & 123.92 \\
\hline \multicolumn{7}{|c|}{$\mathrm{L} 2$ dataset partitioned by age of $\mathrm{L} 2$ acquisition onset } \\
\hline Childhood & 25 & 489 & 0.41 & $0.28,0.53$ & 0.29 & 33.25 \\
\hline Adulthood & 50 & 759 & 0.24 & $0.13,0.34$ & 0.24 & 65.06 \\
\hline \multicolumn{7}{|c|}{ Infant dataset partitioned by English as the L2 } \\
\hline \multicolumn{7}{|c|}{ L2 dataset partitioned by L2 proficiency } \\
\hline Proficient & 87 & 1132 & 0.20 & $0.11,0.28$ & 0.17 & 72.40 \\
\hline Nonproficient & 19 & 455 & 0.39 & $0.26,0.53$ & 0.33 & $45.43^{*}$ \\
\hline \multicolumn{7}{|c|}{ Nonproficient dataset partitioned by English as the L2 } \\
\hline L2 English & 8 & & 0.70 & $0.52,0.89$ & & 10.31 \\
\hline Dual task & 23 & 359 & 0.23 & $0.08,0.37$ & 0.27 & 15.12 \\
\hline \multicolumn{7}{|c|}{ L2 dataset partitioned by linguistic task demands } \\
\hline Single words & 35 & 735 & 0.41 & $0.30,0.51$ & 0.44 & 50.62 \\
\hline Phonological-word pairs & 8 & 48 & 0.07 & $-0.34,0.47$ & 0.06 & 5.99 \\
\hline Orthographic-word pairs & 45 & 532 & 0.11 & $-0.01,0.23$ & 0.10 & 35.12 \\
\hline Semantic-word pairs & 13 & 206 & 0.21 & $0.01,0.40$ & 0.04 & 14.09 \\
\hline
\end{tabular}

Note: ${ }^{*} p<0.05$, d.f. $=k \times 1 ; k=$ number of independent effect sizes; CI: confidence interval; L2: second language. Positive effect sizes $(d \mathrm{~s})$ reflect greater activation in the left hemisphere when the CIs do not include zero and bilateral activation when the CIs include zero. 


\begin{tabular}{|c|c|c|}
\hline Attachment & Stem & Leaf \\
\hline 2 & Extremes $<.72$ & \\
\hline 1 & -.6 & 7 \\
\hline 0 & -.5 & 5 \\
\hline 3 & -.4 & 227 \\
\hline 3 & -.3 & 158 \\
\hline 6 & -.2 & 013589 \\
\hline 8 & -.1 & 01455566 \\
\hline 11 & -.0 & 00112233567 \\
\hline 13 & .0 & 0001555556788 \\
\hline 9 & .1 & 345779999 \\
\hline 11 & .2 & 00001123578 \\
\hline 10 & .3 & 0134558899 \\
\hline 7 & .4 & 0012447 \\
\hline 5 & .5 & 25689 \\
\hline 5 & .6 & 37899 \\
\hline 2 & .7 & 39 \\
\hline 5 & .8 & 00189 \\
\hline 2 & .9 & 23 \\
\hline 1 & 1.0 & 0 \\
\hline 2 & Extremes $>1.11$ & \\
\hline
\end{tabular}

Fig. 2. Stem and leaf display of effect sizes for L2 laterality.

\subsubsection{Second language proficiency}

Categorical modeling of mean L2 effect sizes by L2 proficiency found a moderate LH effect for nonproficient bilinguals $(d=0.39 ; 95 \% \mathrm{CI}=0.26,0.53$; $k=19)$ that was significantly stronger than the LH effect found for proficient bilinguals in $\mathrm{L} 2(d=0.20 ; 95 \% \mathrm{CI}=0.11,0.28 ; k=87)$, as shown by the betweengroups homogeneity statistic, $Q_{\mathrm{B}}(1)=6.09, p<0.05$. This pattern of results mirrors that of the L1 analysis, except that the nonproficient sample here was large enough $(k=19)$ to be statistically reliable. However, homogeneity was not retained within the nonproficient group, $Q_{\mathrm{W}}(18)=45.43, p<0.01$, indicating the operation of an additional moderator.

Categorical modeling of the proficient L2 sample by age of bilingualism onset revealed that proficient infant onset bilinguals were bilaterally organized for language $(d=0.03 ; 95 \% \mathrm{CI}=-0.13,0.18 ; k=29)$, whereas childhood $(d=0.28 ; 95 \% \mathrm{CI}=0.12,0.43 ; k=21)$ and adult onset bilinguals were LH dominant $(d=0.25 ; 95 \% \mathrm{CI}=0.12,0.38 ; k=37)$. Moreover, infant onset bilinguals were significantly less lateralized than both childhood, $Q_{\mathrm{B}}(1)=4.87, p<0.05$, and adult onset bilinguals, $Q_{\mathrm{B}}(1)=4.46, p<0.05$, who did not differ from each other, $Q_{\mathrm{B}}(1)=0.06$, n.s. Categorical modeling of the nonproficient L2 sample by age of bilingualism onset showed that nonproficient adult onset bilinguals were only slightly LH dominant $(d=0.21 ; 95 \% \mathrm{CI}=0.05,0.38 ; k=13)$, but cell sizes were too small to analyze for infant and childhood onset nonproficient bilinguals.

Categorical modeling of the proficient L2 sample by English as the L2 showed no difference in LH laterality whether English $(d=0.20 ; 95 \% \mathrm{CI}=0.10$, $0.31 ; k=49)$ or "other" $(d=0.18 ; 95 \% \mathrm{CI}=0.04,0.32 ; k=38)$ was the $\mathrm{L} 2$, $Q_{\mathrm{B}}(1)=6.92, n$.s. All variance was also explained in the nonproficient L2 sample when partitioned by English as the L2, where English, $Q_{\mathrm{W}}(7)=10.31$, n.s. and other $\mathrm{L} 2 \mathrm{~s}, Q_{\mathrm{W}}(10)=12.97$, n.s., were each internally consistent. However, nonproficient bilinguals with English as the L2 were strongly and significantly more LH lateralized $(d=0.70 ; 95 \% \mathrm{CI}=0.52,0.89 ; k=8)$ than those with other L2s $(d=0.06 ; 95 \% \mathrm{CI}=-0.13,0.26 ; k=11)$, as shown by the significant homogeneity statistic, $Q_{\mathrm{B}}(1)=22.14, p<0.001$. These outcomes provide support for 
the notion of language specific effects on lateralization of function and for the possibility that learning English as L2 may promote greater LH participation than learning other L2s, perhaps as a result of the unusually high incidence of irregularity in spelling-to-sound correspondence in English relative to other languages. Moreover, the present finding for a reduced LH effect for English in proficient relative to nonproficient bilinguals is consistent with the view that, once an $\mathrm{L} 2$ becomes automatized, less reliance on a frontal $\mathrm{LH}$ system is required (Ullman, 2001).

\subsubsection{Age of bilingualism onset}

Categorical modeling of L2 effect size variance by age of bilingualism onset indicated that adult and childhood onset bilinguals were significantly LH dominant for $\mathrm{L} 2(d=0.24 ; 95 \% \mathrm{CI}=0.13,0.34 ; k=50$, and $d=0.41 ; 95 \%$ $\mathrm{CI}=0.28,0.53 ; k=25$, respectively), and both groups retained homogeneity, $Q_{\mathrm{W}}(49)=65.06, n . s ., Q_{\mathrm{W}}(24)=33.25$, n.s., respectively. However, whereas first language laterality did not differ between bilinguals who acquired the $\mathrm{L} 2$ during childhood versus adulthood, childhood onset bilinguals were significantly more LH lateralized than adult onset bilinguals for the second language, $Q_{\mathrm{B}}(1)=4.10$, $p<0.05$. One possible reason for the relatively increased $\mathrm{LH}$ role in childhood bilinguals is that $\mathrm{L} 2$ learning during childhood is often undertaken in a formal, classroom setting that is quite rule-based and grammar intensive, and may thus promote an over reliance on LH strategies for language processing in general.

For bilinguals who acquired the L2 during infancy, a bilateral effect for L2 was again revealed $(d=0.08 ; 95 \% \mathrm{CI}=-0.08,0.22 ; k=31)$. The $\mathrm{L} 2$ analysis further showed the infant onset group to be significantly less LH lateralized than the childhood onset group, $Q_{\mathrm{B}}(1)=11.16, p<0.01$, and marginally less so than the adult onset group, $Q_{\mathrm{B}}(1)=3.28, p<0.07$. However, homogeneity for infant onset bilinguals was not retained in the L2 analysis, $Q_{\mathrm{W}}(30)=14.29, p<0.05$. Given that models of L2 proficiency were moderated by age of bilingualism onset, categorical modeling of infant onset bilinguals by L2 proficiency was pursued but rejected because of small cell sizes. However, as with L 2 proficiency, categorical modeling of age of bilingualism onset by English as the L2 was successful in explaining all effect size variance.

\subsubsection{English as the L2}

As was the case for nonproficient L2 groups separately analyzed according to whether English was the L2, all effect size variance was explained in the infant onset L2 group when English, $Q_{\mathrm{W}}(11)=6.94, p=0.86$, or "other" was the $\mathrm{L} 2, Q_{\mathrm{W}}(18)=5.29, p=0.99$. Specifically, while both infant onset groups were bilaterally activated for language, L2s other than English $(d=-0.02 ; 95 \%$ $\mathrm{CI}=-0.24,0.21 ; k=17)$ tended to show less LH involvement than English $(d=0.32 ; 95 \% \mathrm{CI}=0.23,0.41 ; k=31)$, though the difference did not reach significance, $Q_{\mathrm{B}}(1)=1.01, p=0.31$. Notably, the same hierarchical model (i.e., English versus "other" nested within infant L2 acquisition age) was also successful in explaining effect size variance within L1s.

\subsubsection{Language component}

In general, results of the L 2 analysis for language components mirrored those of the L1 analysis. Like the L1 analysis, outcomes from categorical models of syntactic word pair judgments and sentence comprehension were each based on fewer than five data points and thus are not discussed further.

The L2 analysis for language components revealed a bilateral effect for orthographic processing $(d=0.11 ; 95 \% \mathrm{CI}=-0.01,0.23 ; k=45)$ and a very small LH effect for semantic processing $(d=0.21 ; 95 \% \mathrm{CI}=0.01,0.40 ; k=13)$. Homogeneity was retained within both the semantic, $Q_{\mathrm{W}}(12)=14.09$, n.s., and orthographic divisions, $Q_{\mathrm{W}}(44)=35.12$, n.s. It is interesting that L1 semantic processing was bilateral and L1 orthographic processing was weakly LH dominant, the reverse of the L2 pattern. However, direct contrasts showed no differences between $\mathrm{L} 1$ and $\mathrm{L} 2$ laterality for either the semantic, $Q_{\mathrm{B}}(1)=0.02$, n.s., or the orthographic divisions, $Q_{\mathrm{B}}(1)=0.07$, n.s. Thus, outcomes from the L1 and L2 analyses of orthographic and semantic language components are consistent with each other and with predictions in the literature for reduced $\mathrm{LH}$ dominance for these components (e.g., Vaid, 1984a). Also like the L1 analysis, the L2 analysis supported predictions in the literature (e.g., Chiarello et al., 2002) by revealing a robust and reliable LH effect for single word responses $(d=0.41 ; 95 \% \mathrm{CI}=0.30,0.51 ; k=35)$, and homogeneity was retained, $Q_{\mathrm{W}}(34)=50.62, n . s$.
The categorical model for phonological features of L2 words indicated bilateral symmetry in bilinguals $(d=0.07 ; 95 \% \mathrm{CI}=-0.34,0.47 ; k=8)$, and homogeneity was retained, $Q_{\mathrm{W}}(7)=5.99, p=0.65$. Taken together with similar results from the L1 meta-analysis, this provides reliable evidence that phonological processing in bilinguals is bilaterally mediated, at least at the word level, and is consistent with current imaging findings for a decrease in LH dominance during speech perception (e.g., Hickok \& Poeppel, 2004).

\subsubsection{Experimental paradigm}

The L2 categorical model for experimental paradigm generally replicated the L1 outcomes. Specifically, there was a relatively strong LH effect for L2 processing in the dichotic listening paradigm $(d=0.45 ; 95 \% \mathrm{CI}=0.33,0.58$; $k=23)$ and a weak LH effect for the visual paradigm $(d=0.11 ; 95 \% \mathrm{CI}=0.00$, $0.21 ; k=60)$. However, the $\mathrm{L} 2$ analysis also showed a small $\mathrm{LH}$ effect for the dual task division $(d=0.23 ; 95 \% \mathrm{CI}=0.08,0.37 ; k=23)$, whereas the $\mathrm{L} 1$ model showed bilateral activation. Direct contrasts showed no difference in laterality for dual task paradigms between L1s and L2s, $Q_{\mathrm{B}}(1)=0.06$, n.s. Thus, consistent outcomes emerged across the two analyses for all experimental paradigms, indicating that language laterality results are influenced by paradigm choice.

A potential concern is that the finding for decreased LH laterality in infant onset bilinguals relative to those who learn an L2 later may simply reflect a disproportionate influence of the dichotic listening paradigm among late bilinguals. To investigate this concern, inspection of laterality effects was carried out for each age of onset within each paradigm, regardless of cell size (indicated by the value of $k$ provided for each cell).

For dichotic listening in L1, infant onset bilinguals were bilaterally activated and homogenous ( $d=-0.02 ; 95 \% \mathrm{CI}=-1.00,0.97 ; k=3), Q_{\mathrm{W}}(2)=0.61$, n.s.; childhood onset bilinguals were strongly LH dominant but not homogenous ( $d=0.51 ; 95 \% \mathrm{CI}=0.28,0.74 ; k=6), Q_{\mathrm{W}}(5)=23.69, p<0.01$; and adult onset bilinguals were moderately LH dominant and homogenous $(d=0.35 ; 95 \%$ $\mathrm{CI}=0.17,0.53 ; k=9), Q_{\mathrm{W}}(8)=11.80, n . s$. For the visual paradigm in L1, infant onset bilinguals were bilaterally activated and homogenous $(d=-0.02 ; 95 \%$ $\mathrm{CI}=-0.22,0.18 ; k=21), Q_{\mathrm{W}}(20)=10.71$, n.s.; childhood onset bilinguals were bilaterally activated and homogenous $(d=0.20 ; 95 \% \mathrm{CI}=-0.03,0.44 ; k=8)$, $Q_{\mathrm{W}}(7)=11.88$, n.s.; adult onset bilinguals were LH dominant and homogenous $(d=0.34 ; 95 \% \mathrm{CI}=0.13,0.54 ; k=26), Q_{\mathrm{W}}(25)=18.95$, n.s. For the dual task paradigm in L1, infant onset bilinguals were bilaterally activated and homogenous $(d=0.00 ; 95 \% \mathrm{CI}=-0.25,0.25 ; k=7), Q_{\mathrm{W}}(6)=1.38, n . s$. childhood onset bilinguals were LH dominant and homogenous $(d=0.22 ; 95 \% \mathrm{CI}=0.01,0.44$; $k=12), Q_{\mathrm{W}}(11)=4.57$, n.s.; adult onset bilinguals were bilaterally activated but not homogenous $(d=0.10 ; 95 \% \mathrm{CI}=-0.16,0.36 ; k=6), Q_{\mathrm{W}}(5)=18.79$, $p<0.01$.

For dichotic listening in $\mathrm{L} 2$, infant onset bilinguals were bilaterally activated and homogenous ( $d=-0.09 ; 95 \% \mathrm{CI}=-0.57,0.38 ; k=5), Q_{\mathrm{W}}(4)=0.48$, n.s.; childhood onset bilinguals were strongly LH dominant and homogenous $(d=0.65 ; 95 \% \mathrm{CI}=0.45,0.85 ; k=6), Q_{\mathrm{W}}(5)=6.45$, n.s.; and adult onset bilinguals were moderately LH dominant and homogenous $(d=0.38 ; 95 \%$ $\mathrm{CI}=0.22,0.55 ; k=12), Q_{\mathrm{W}}(8)=22.48, p<0.05$. For the visual paradigm in $\mathrm{L} 2$, infant onset bilinguals were bilaterally activated and homogenous $(d=0.09$; $95 \% \mathrm{CI}=-0.09,0.27 ; k=22), Q_{\mathrm{W}}(21)=13.22$, n.s.; childhood onset bilinguals were bilaterally activated and homogenous $(d=0.23 ; 95 \% \mathrm{CI}=-0.00$, $0.47 ; k=8), Q_{\mathrm{W}}(7)=12.41$, n.s.; adult onset bilinguals were bilaterally activated and homogenous $(d=0.06 ; 95 \% \mathrm{CI}=-0.10,0.23 ; k=30), Q_{\mathrm{W}}(29)=23.09$, n.s. Finally, for the dual task paradigm in $\mathrm{L} 2$, infant onset bilinguals were bilaterally activated and homogenous $(d=0.07 ; 95 \% \mathrm{CI}=-0.32,0.45 ; k=4)$, $Q_{\mathrm{W}}(3)=0.13$, n.s.; childhood onset bilinguals were bilaterally activated and homogenous $(d=0.22 ; 95 \% \mathrm{CI}=-0.02,0.45 ; k=11), Q_{\mathrm{W}}(10)=4.11, n . s . ;$ adult onset bilinguals were LH dominant and homogenous $(d=0.28 ; 95 \% \mathrm{CI}=0.06$, $0.50 ; k=8), Q_{\mathrm{W}}(7)=9.97$, n.s.

These outcomes make clear that infant onset bilinguals were bilaterally activated for both languages within each paradigm, including the dichotic listening paradigm, and that they consistently showed less LH involvement for language tasks than both childhood and adult onset bilinguals regardless of paradigm. In contrast, childhood and adult onset bilinguals were always LH dominant for the dichotic listening paradigm and often for other paradigms as well. Therefore, the distribution of paradigm types across bilingual subgroups was not a significant factor in differential laterality outcomes, at least with respect to functional lateralization. 
Table 5

Summary of mean effect size comparisons for L1 vs. L2

\begin{tabular}{lcll}
\hline Moderator division for L1 vs. L2 comparison & Number of comparisons $C$ & Fit statistic $Q_{\mathrm{B}}$ & Interpretation of L1 vs. L2 comparisons \\
\hline Bilingualism onset in infancy & 31 & 0.52 & No difference for infant bilinguals \\
Bilingualism onset in childhood & 25 & 1.03 & No difference for childhood bilinguals \\
Bilingualism onset in adulthood & 41 & 0.54 & No difference for adolescent bilinguals \\
Proficiency in L2 & 87 & 0.62 & No difference for fluent bilinguals \\
Nonproficiency in L2 & 6 & $5.44^{*}$ & Greater LH in L1 than L2 \\
Visual paradigm & 56 & 0.55 & No difference in visual paradigms \\
Dichotic listening paradigm & 17 & 0.26 & No difference in dichotic listening \\
Dual task paradigm & 23 & 0.98 & No difference in dual task paradigms \\
Responses to single words & 28 & 0.85 & No difference in single words \\
Phonological word judgments & 8 & 0.78 & No difference in phonological judgments \\
Orthographic word judgments & 43 & 0.81 & No difference for orthographic judgments \\
Semantic judgments, word pairs & 9 & 0.02 & No difference for semantic judgments \\
English as L2 & 52 & 0.34 & No difference for English as L2 \\
Other as L2 & 46 & 0.01 & No difference for other as L2 \\
\hline
\end{tabular}

Note: ${ }^{*} p<0.05$, d.f. $=C-1 ; C=$ number of comparisons.

\subsection{Results and discussion of comparisons of L1 and L2 laterality}

The findings from the separate meta-analyses of L1 and L2 make at least two points that are critical to theories concerning organization of languages in the bilingual brain. First, whereas interactions among moderators are necessary to explain all variance within bilingual groups for $\mathrm{L} 1$ and for $\mathrm{L} 2$, all explanatory models consistently reveal similar patterns of bilateral activation in infant onset bilinguals and LH dominance in general for childhood and adult onset bilinguals. Second, the two meta-analyses provide strong evidence that the various components of language tested here engage similar patterns of functional language laterality across L1 and L2, suggesting that the two languages of bilinguals may not be functionally distinct.

To address whether L1 and L2 are separate language entities, or at least are functionally organized as such, direct contrasts were carried out on the mean effect sizes for each division of every moderating variable that was coded in the present research. The mean effect sizes for L1 and for L2 for each moderator division (e.g., single words) were collapsed and the homogeneity statistic was computed. A significant homogeneity statistic would indicate that L1 and L2 effect sizes derived from different populations, whereas a nonsignificant homogeneity statistic would indicate that L1 and L2 effect sizes were internally consistent. The outcomes clearly showed there was no difference for L1 and L2 effect sizes within each bilingualism onset age group, within each paradigm, within proficient bilinguals, or within language components tested (see Table 5). Furthermore, whereas L1 was more LH lateralized than L2 for nonproficient (late) bilinguals, both languages were LH lateralized, consistent with current theories in the literature that predict both languages of late, proficient bilinguals will be LH dominant (Ullman, 2001) and thus could be supported by convergent neural substrates (Green, 2003).

In summary, our findings suggest that although the pattern of language lateralization differs between bilingual subgroups, within bilinguals the two languages show functionally very similar patterns. Moreover, the pattern of laterality within each bilingual subgroup is reliable across languages. That is, when one language of infant onset bilinguals is bilaterally organized, so is the other. Likewise, when the L1 of late bilinguals is LH dominant, so is the L2. Indeed, L1 and L2 laterality patterns within each group are comparable enough to provide a persuasive argument that the functional lateralization of first language(s) that is set up in the brain during early development may anchor the organization of subsequently learned languages (henceforth, the Anchoring Hypothesis).

Our Anchoring Hypothesis would suggest that third languages (and fourth, and so on) should follow the same pattern uncovered here for second languages. Whereas we were unable to unearth appropriate behavioral laterality data from multilinguals to formally test this idea, emerging neuroimaging data are consistent with this prediction. For example, Vingerhoets et al. (2003) used whole-head fMRI to specifically test whether the different languages in proficient, late-onset trilinguals (Dutch, French, and English) were represented in different brain regions. The outcomes revealed very similar patterns of extensive LH activation for all three languages during language production as well as comprehension tasks. In addition, generally increased LH activation (particularly in the left posterior temporal region) was noted for the second and third languages relative to L1. The authors concluded that largely the same neural substrates were involved in processing all three of the trilinguals' languages and that additional activation associated with the foreign languages may have been a result of somewhat lesser proficiency for $\mathrm{L} 2$ and $\mathrm{L} 3$ relative to $\mathrm{L} 1$.

Perhaps the strongest test of our Anchoring Hypothesis can be found in another recent imaging study that included proficient quadrilinguals (Briellmann et al., 2004). Of particular relevance is that one of the quadrilinguals learned two languages from infancy but learned the other two later, whereas the remaining five quadrilinguals learned only one native language but learned the other three as foreign languages during childhood or later. In addition to the variations in multilingualism onset ages, the participants varied in terms of foreign language proficiency, although all were highly proficient in at least two of their four languages. Thus, the variations in multilingualism onset-ages among participants in the Briellmann et al. study provide a direct test of the notion that the organization of native language(s) influences that of foreign languages while also taking into account any influence of language proficiency.

Using fMRI to measure neural activity during covert word production tasks in a variety of languages (i.e., English, German, Italian, French, and/or Spanish, depending on participants' knowledge of each), Briellmann et al. (2004) found largely overlapping activation across languages for each participant, indicating that the same neural substrates supported all four languages within individuals. Moreover, although hemispheric dominance was not specifically measured, examination of the representative slices for each individual clearly shows that the late multilinguals showed more $\mathrm{LH}$ (relative to $\mathrm{RH}$ ) activation overall, and all but one showed increasing amounts of activation as proficiency in the foreign languages decreased. Conversely, the infant-onset multilingual showed virtually identical bilateral activation for all four languages, including the nonproficient language. These outcomes are highly consistent with the present meta-analytic outcomes and with our Anchoring Hypothesis.

The present research gave particular attention to comparing and contrasting the explanatory value of the Age Hypothesis and the Stage Hypothesis. The results indicate that age of bilingualism onset (Age Hypothesis) is superior to L2 proficiency (Stage Hypothesis) as a predictor of bilingual language laterality. Specifically, the effects of bilingualism onset age remain unchanged when modeled by L2 proficiency, experimental paradigm, English as the L2, and language component. In contrast, categorical modeling of L2 proficiency by bilingualism onset age actually changes the outcomes of the L2 proficiency model.

The Stage Hypothesis is admittedly difficult to test with infant onset bilinguals. Although the two data points from nonproficient infant onset bilinguals in the present sample did show bilateral effects, the very fact that there were only two such sources among the 2000 bilinguals tested makes clear that infant 
onset bilinguals are typically proficient in both languages. Moreover, the reliable finding of bilateral organization for infant onset bilinguals (regardless of proficiency), as opposed to a different finding for proficient childhood and adult onset bilinguals, namely, LH dominance, undermines the value of L 2 proficiency alone as reliable predictor of laterality across bilingual language experience groups. Nevertheless, an important role for the influence of L2 proficiency on laterality for late bilinguals is clear, although in a direction that is opposite to that initially introduced in the Stage Hypothesis. That is, the present outcomes showed that less proficiency in the L2 was associated with significantly more LH (rather than $\mathrm{RH}$ ) involvement for both L1 and L2 in late bilinguals. We suggest that L2 proficiency is best indicated for predictions concerning the laterality of late-onset bilinguals, i.e., those who acquired the L2 well after the L1.

\section{General discussion}

The present research consisted of two meta-analyses that assessed bilingual language laterality in L1 and L2. The primary aim was to test the primacy of two competing variables in this literature, namely, the age of second language acquisition and the proficiency of second language acquisition. Whereas the present research shows a robust effect of acquisition age on language laterality regardless of proficiency, the direction of the difference is for increased RH involvement in early relative to late bilinguals, in contrast with initial formulations of the Age Hypothesis that predicted increased RH involvement for late bilinguals relative to monolinguals and early bilinguals. Thus, the Age Hypothesis is supported specifically in terms of its present operationalization, i.e., that brain maturational and cognitive differences will give rise to differential functional organization of languages acquired at different points during development. Whereas evidence garnered from behavioral laterality studies is inferential and cannot directly point to underlying neural mechanisms, our findings indicate that, at least at a functional level, language representation in the brain differs between early and late bilinguals, even allowing for individual variation in L2 mastery. These findings corroborate those of a previous meta-analysis that also found reliable patterns of bilateral activation for infant onset bilinguals and LH dominance for late onset bilinguals in their L1s (Hull \& Vaid, 2006).

One possible explanation for the relatively greater RH involvement in infant onset bilinguals is the pragmatic necessity of developing and applying metalinguistic knowledge from a very early age in order to monitor the language environment and use the appropriate language in certain situations and/or with certain people (Bialystok, 2001; Genesee, 2001; see also Long, 1990). This possibility is consistent with recent evidence that patients with RH damage are deficient in using pragmatic cues to understand and produce language discourse (Cheang \& Pell, 2006). Another compelling possibility has been offered by Sebastian-Galles and Bosch (2005), who argue that different aspects of language processing, such as phonology and grammar, could well be differentially sensitive to age of bilingualism onset and L2 proficiency, an idea which, if borne out, will go far in organizing the diversity of outcomes across not only behavioral bilingual laterality studies but also functional neuroimaging studies of bilinguals (see also Wartenburger et al., 2003). Moreover, this argument is consistent with the ubiquitous but as yet incompletely understood finding for superior grammatical processing in early versus late proficient bilinguals who nevertheless show equivalent phonological processing (see Birdsong, 2005).

Another possible source of differential hemispheric involvement in early versus late bilinguals is that the sheer amount of experience early bilinguals have with handling two language systems may intensify or accelerate the automatization of language processes, and may thereby transfer those processes to different functional and/or structural pathways than those of less experienced bilinguals, even proficient ones (see Indefrey, 2006; Raichle et al., 1994; Sebastian-Galles \& Bosch, 2005). However, to disentangle effects of length of language exposure and age of language acquisition would necessitate cross-sectional studies (e.g., comparing early bilingual children with late bilingual adults), which would in turn introduce yet another potential confound in terms of age differences in the comparison groups. It may be that a definitive answer to potential effects of length of language exposure on language laterality cannot be reached, and certainly we were not able to specifically test for it here, because none of the studies assessed that variable. Nonetheless, although it is not possible to rule out a potential influence of length of language exposure on our finding for increased $\mathrm{RH}$ dominance in infant-onset relative to late-onset bilinguals, it remains that the functional lateralization of language is particularly dependent on early language experience.

The present research did not find support for the Stage Hypothesis, which predicts greater RH participation in nonproficient relative to proficient bilinguals. Quite the contrary, the outcomes demonstrate that nonproficient bilinguals are actually more LH dominant than proficient bilinguals in both L1 and L2, corroborating findings from a previous meta-analysis of monolingual versus bilingual language laterality (Hull \& Vaid, 2006) and from recent neuroimaging evidence, which suggests that one likely reason for increased LH participation is that more processing effort (and thus more extensive neural activation) is required to engage the language that is less proficient in lateonset bilinguals (Briellmann et al., 2004; Vingerhoets et al., 2003).

While behavioral measures of hemispheric laterality are indirect indices of neural activity, they do suggest important generalities regarding brain organization of language, namely, that the patterns of functional hemispheric involvement set up during L1 learning also underlie L2 processing. In the case of infant onset bilinguals, for whom both languages may be considered L1, the simultaneous learning of two languages appears to generate a unique pattern of bilateral language organization in the brain. Conversely, bilinguals who acquire only one language during early development appear to functionally organize language in the LH, just as monolinguals do, and this pattern remains stable even when an L2 is acquired later on.

If the present finding of a substantial overlap in L1 and L2 functional representation comes as a surprise, perhaps it is because many approaches to bilingual linguistic organization have assumed a functional separation between L1 and L2 lexicons or linguistic systems in general. This notion may have been influenced by suggestions of differential language representation discussed in the aphasia literature based on findings of 
differential impairment and recovery for L1 and L2 in bilinguals. However, even within the aphasia literature it has been argued that any notion of differential localization of the bilingual's languages is speculative at best, and unsupported by the majority of cases observed. Nevertheless, the notion of functional separation of the bilinguals' two languages has a strong following in current psycholinguistic models of the bilingual mental lexicon, which assume that the two languages are represented in separate and at least partially independent lexicons (e.g., Dufour \& Kroll, 1995; Kroll \& Stewart, 1994; for reviews see Dijkstra \& Van Heuven, 2002; Francis, 2005).

The idea that a bilingual's two languages are functionally separate may also have been reinforced by a much-cited fMRI report of a functional separation of L1- and L2-associated activity in the left inferior frontal cortex, or Broca's area (Kim, Relkin, Lee, \& Hirsch, 1997). However, there are questions as to the appropriateness of the analyses used in that study. In particular, the inferential analyses reported in the Kim et al. study treated activation in Broca's and Wernicke's areas as independent within individuals (Francis, 2003). When Francis recomputed the same measures using inferential statistics for dependent samples on the data for late bilinguals as reported in the Kim et al. study, the effects did not reach significance; specifically, no significant differences emerged in the $t$-test comparison of the differences between centroid distances in Broca's versus Wernicke's areas $(p>0.4)$, there was no main effect in the analysis of variance for cortical region (Broca's versus Wernicke's; $p=0.091$ ), nor was there an interaction of bilingual type (early versus late) with cortical region $(p=0.086)$.

Concomitant with advances in the precision of neuroimaging measurement and analysis techniques since the appearance of the Kim et al. (1997) study, a number of more recent PET and fMRI studies have demonstrated that the prevailing pattern is one of overlapping rather than separate neural circuitry underlying L1 and L2 performance, both for single word paradigms (Chee, Tan, \& Thiel, 1999a; Hernandez, Martinez, \& Kohnert, 2000; Illes et al., 1999; Klein, Milner, Zatorre, Zhao, \& Nikelski, 1999) and for tasks engaging sentence and discourse level processing (Briellmann et al., 2004; Chee, Tan, \& Thiel, 1999b; Perani et al., 1996; Vingerhoets et al., 2003). The increasing prevalence of findings that L1 and L2 overlap over a range of neurobehavioral studies with different participant and task variables (see Indefrey, 2006) is consistent with the present research outcomes. Taken together, these outcomes support the notion of a convergence model of bilingual lexical representation, at least for bilinguals who are proficient in both languages (Chee et al., 1999b; Green, 2003).

It should be noted that the functional overlap for L1 and L2 bilingual laterality found in the present research and in many neuroimaging studies is in opposition to psycholinguistic models that assume a differential organization of L1 and L2 as well as accounts based on the polyglot aphasia or the electrocortical stimulation literatures (see Vaid, in press). It is critical to note, however, that most studies in the psycholinguistic literature on bilingualism have drawn primarily on data from late bilinguals. As a result, few studies have explicitly considered the influence of early versus late language acquisition on the functional architecture of the bilingual mental lexicon. Therefore, if we only consider data from proficient, late bilinguals in the present meta-analysis, the finding for similar lateralization of L1 and L2 is consistent with existing models of neurofunctional organization of grammar and the lexicon that are based on language proficiency. In particular, both the declarative/procedural (Ullman, 2001) and the convergence models (Green, 2003) predict that proficient L1s and L2s will be similarly LH lateralized, and this prediction is supported in the present results.

Theoretical accounts based on lesion data showing differential recovery from aphasia for L1 and L2 may also be reconciled with the present results. Specifically, the idea that the bilingual is able to selectively activate or inhibit a given language (e.g., Green, 1998, 2005) allows for a functional explanation for the diverse patterns of recovery observed in bilingual aphasics in terms of variations in damage to the bilingual's inhibitory mechanism rather than to some language module or other neural structure devoted to the interrupted language. This idea fits well with the present findings that the two languages of bilinguals are lateralized similarly for verbal tasks.

A functional explanation also sheds new light on some otherwise difficult to explain patterns of language recovery, such as the ability of some aphasics to speak proficiently in one language but not the other one day, but to show the reverse pattern the next day (Paradis, 2000). Patterns such as these are consistent with the notion of a disruption of control over the activation or suppression of the languages. In sum, a functional explanation for an overlapping language representation system in the bilingual brain, as found here, provides an alternative to the pervasive idea that nonparallel language recovery must mean that different parts of the brain are responsible for processing the different languages of bilinguals (see also Meuter, 2005).

Other arguments in favor of a functional overlap in L1 and L2 that are consistent with the present findings include Grosjean's (1989) proposal that the two languages of bilinguals work as a single, integrated system in which the individual can choose to operate in either a bilingual mode that keeps both languages available, or in a monolingual mode that actively selects one language and deactivates the other (see also Grosjean, Li, Munte, \& Rodriguez-Fornells, 2003; Green, 2005). The bi-directional transfer view (Pavlenko \& Jarvis, 2002) also advocates bilinguals as integrated users of their two languages (i.e., that functioning in one language is not independent of functioning in the other). Pavlenko and Jarvis showed that transfer of lexical and semantic information can proceed from L1 to L2, as well as from L2 to L1, even in individuals with less proficiency in the L2 (see also Cook, 2003). Finally, Dijkstra and Van Heuven (2002) have provided evidence for nonselective language access, i.e., that both languages of bilinguals are activated during verbal processing, even when input and output are confined to a single language.

\section{Conclusions}

The present research has provided a critical test of two prominent hypotheses concerning functional lateralization of language in the bilingual brain. Our results indicate that age of onset of 
bilingualism is an important variable with a clear and consistent effect on lateralization, whereas the effect of proficiency is relatively limited. More generally, our research demonstrates that the behavioral bilingual laterality literature is, in fact, interpretable, given that reliable outcomes are obtained when relevant moderators are controlled. The present findings also point to the importance of basing empirical research questions on theoretically relevant issues and designing studies that take into account the particular individual difference and task variables shown to systematically underlie variability among bilinguals. As such, consideration of the behavioral language laterality literature may offer valuable lessons to the fast-developing neurobehavioral field (see also Hull \& Vaid, 2005).

Finally, and perhaps most importantly, our finding that early bilinguals are bilaterally organized for language substantiates, on a larger corpus, a similar conclusion arrived at in an early meta-analysis of bilinguals (Vaid \& Hall, 1991) and in a recent meta-analysis that compared bilinguals with monolinguals (Hull \& Vaid, 2006). This initially unexpected but consistent finding of bilaterality associated with early multiple language experience challenges the normative view of the $\mathrm{LH}$ as the dominant language hemisphere. Given that this view has been based on a subset of the world's language users (i.e., those with a single, initial language), it would appear that a rethinking of what constitutes the norm in the study of language and the brain may be warranted.

\section{Acknowledgements}

We thank Robert Hudson and Jennifer Wilkins for their assistance in coding the primary studies, and Morris Moscovitch for insightful comments on a previous version of this paper. We are grateful to a number of investigators for providing us with copies of their research in the cases where it is as yet unpublished.

\section{References $^{2}$}

Abutalebi, J., Cappa, S., \& Perani, D. (2005). What can functional neuroimaging tell us about the bilingual brain? In J. Kroll \& A. M. B. deGroot (Eds.), Handbook of bilingualism: Psycholinguistic approaches (pp. 497-515). London: Oxford University Press.

*Albanese, J. (1985). Language lateralization in English-French bilinguals. Brain and Language, 24, 284-296.

-Albert, M., \& Obler, L. K. (1978). The bilingual brain: Neuropsychological and neurolinguistic aspects of bilingualism. New York: Academic Press., pp. 157-201.

Arthur, W., Bennett, W., \& Huffcutt, A. (2001). Conducting meta-analysis using SAS. Mahwah, NJ: Lawrence Erlbaum Associates.

*Bentin, S. (1981). On the representation of a second language in the cerebral hemispheres of right-handed people. Neuropsychologia, 19(4), 599-603.

-Bergh, G. (1986). The neuropsychological status of Swedish-English subsidiary bilinguals. Gothenburg Studies in English, 61, 31-65.

\footnotetext{
${ }^{2}$ References marked with an asterisk indicate studies included in the metaanalyses. References marked with a dash indicate studies that provided L1 and L2 comparison data only.
}

Bialystok, E. (2001). Bilingualism in development: Language, literacy, and cognition. Cambridge, UK: Cambridge University Press.

Birdsong, D. (2005). Interpreting age effects in second language acquisition. In J. Kroll \& A. M. B. deGroot (Eds.), Handbook of bilingualism: Psycholinguistic approaches (pp. 109-127). London: Oxford University Press.

Birdsong, D., \& Molis, M. (2001). On the evidence for maturational constraints in second-language acquisition. Journal of Memory and Language, $44,235-250$

Boles, D. B. (2005). A large-sample study of sex differences in functional cerebral lateralization. Journal of Clinical and Experimental Neuropsychology, $27,759-768$

Briellmann, R. S., Saling, M. M., Connell, A. B., Waites, A. B., Abbott, D. F., \& Jackson, G. D. (2004). A high-yield functional MRI study of quadric-lingual subjects. Brain and Language, 89, 531-542.

-Carroll, F. (1980). Neurolinguistic processing of a second language: Experimental evidence. In R. Scarcella \& S. Krashen (Eds.), Research in second language acquisition (pp. 81-86). Rowley, MA: Newbury House.

Cheang, H., \& Pell, M. (2006). A study of humour and communicative intention following right hemisphere stroke. Clinical Linguistics and Phonetics, 20, 447-462.

Chee, M., Tan, E., \& Thiel, V. (1999a). Mandarin and English single word processing studied with functional magnetic resonance imaging. The Journal of Neuroscience, 19, 3050-3056.

Chee, M., Tan, E., \& Thiel, V. (1999b). Processing of visually presented sentences in Mandarin and English studied with fMRI. Neuron, 23(1), $127-$ 137.

*Chengappa, S., \& Ray, J. (2002). Hemispheric processing of concrete nouns in Kannada monolinguals and Kannada-English bilingual children and adults. Unpublished manuscript. Mysore, India: All India Institute of Speech and Hearing.

Chiarello, C., Liu, S., Shears, C., \& Kacinik, N. (2002). Differential asymmetries for recognizing nouns and verbs: Where are they? Neuropsychology, 16, $35-48$.

Cohen, J. (1992). A power primer. Psychological Bulletin, 112, 155-159.

Cook, V. (Ed.). (2003). Effects of the second language on the first. Clevedon: Multilingual Matters.

Cooper, H., \& Hedges, L. V. (1994). The handbook of research synthesis. New York: Russell Sage Foundation.

Davidson, R., \& Hugdahl, K. (1996). Baseline asymmetries in brain electrical activity predict dichotic listening performance. Neuropsychology, 10(2), 241-246.

DeKeyser, R., \& Larson-Hall, J. (2005). What does the critical period really mean? In J. Kroll \& A. M. B. deGroot (Eds.), Handbook of bilingualism: Psycholinguistic approaches (pp. 88-108). London: Oxford University Press.

Dijkstra, A., \& Van Heuven, W. J. B. (2002). The architecture of the bilingual word recognition system: From identification to decision. Bilingualism: Language and Cognition, 5, 175-197.

Dufour, R., \& Kroll, J. (1995). Matching words to concepts in two languages: A test of the concept mediation model of bilingual representation. Memory and Cognition, 23, 166-180.

*Endo, M., Shimizu, A., \& Nakamura, I. (1981a). Laterality differences in recognition of Japanese and Hangul words by monolinguals and bilinguals. Cortex, $17,391-400$.

*Endo, M., Shimizu, A., \& Nakamura, I. (1981b). The influence of Hangul learning upon laterality differences in Hangul word recognition by native Japanese subjects. Brain and Language, 14, 114-119.

*Fabbro, F. (1992). Cerebral lateralization of human languages: Clinical and experimental data. In J. Wind, C. Chiarello, \& B. Bichakjian (Eds.), Language origin: A multidisciplinary approach (pp. 195-224). London: Kluwer Academic.

Fabbro, F. (2001). The bilingual brain: Cerebral representation of languages. Brain and Language, 79, 211-222.

*Fabbro, F., Gran, L., Basso, G., \& Bava, A. (1990). Cerebral lateralization in simultaneous interpretation. Brain and Language, 39, 69-89.

*Fabbro, F., Gran, L., \& Bava, A. (1988). Modification in cerebral lateralization during the acquisition of a second language (English) in adult Italianspeaking females: An experimental dichotic listening study. In A. Tartabini 
\& M. L. Genta (Eds.), Perspectives in the study of primates (pp. 76-85). Cosenza, Italy: DeRose.

Fabbro, F., Gran, B., \& Gran, L. (1991). Hemispheric specialization for semantic and syntactic components of language in simultaneous interpreters. Brain and Language, $41,1-42$.

Franceschini, R., Zappatore, E., \& Nitsch, C. (2003). Lexicon in the brain: What neurobiology has to say about languages. In J. Cenoz, B. Hufeisen, \& U. Jessner (Eds.), The multilingual lexicon (pp. 153-166). Dordrecht: Kluwer Academic.

Francis, W. S. (2003). Discussion of colloquium, panel on recent investigations of the bilingual brain. Presented at the 4th international symposium on bilingualism, Tempe, AZ.

Francis, W. (2005). Bilingual semantic and conceptual representation. In J. Kroll \& A. M. B. deGroot (Eds.), Handbook of bilingualism: Psycholinguistic approaches (pp. 251-267). London: Oxford University Press.

*Furtado, J., \& Webster, W. (1991). Concurrent language and motor performance in bilinguals: A test of the age of acquisition hypothesis. Canadian Journal of Psychology, 45, 448-461.

Galloway, L., \& Krashen, S. (1980). Cerebral organization in bilingualism and second language. In R. Scarcella \& S. Krashen (Eds.), Research in second language acquisition. Rowley, MA: Newbury House.

-Galloway, L., \& Scarcella, R. (1982). Cerebral organization in adult second language acquisition: Is the right hemisphere more involved? Brain and Language, 16, 56-60.

Genesee, F. (2001). Bilingual first language acquisition: Exploring the limits of the language faculty. Annual Review of Applied Linguistics, 21, 153168.

Genesee, F., Hamers, J., Lambert, W. E., Mononen, L., Seitz, M., \& Starck, R. (1978). Language processing strategies in bilinguals: A neuropsychological study. Brain and Language, 5, 1-12.

Golestani, N., \& Zatorre, R. (2004). Learning new sounds of speech: Reallocation of neural substrates. Neurolmage, 21, 494-506.

Goral, M., Levy, E., \& Obler, L. K. (2002). Neurolinguistic aspects of bilingualism. The International Journal of Bilingualism, 6, 411-440.

*Green, A. (1986). A time-sharing cross-sectional study of monolinguals and bilinguals at different levels of second language acquisition. Brain and $\mathrm{Cog}$ nition, 5, 477-497.

Green, D. (1998). Mental control of the bilingual lexico-semantic system. Bilingualism: Language and Cognition, 1, 67-81.

Green, D. (2003). Neural basis of lexicon and grammar in L2 acquisition: The convergence hypothesis. In R. van Hout, A. Hulk, F. Kuiken, \& R. Towell (Eds.), The interface between syntax and the lexicon in second language acquisition (pp. 197-208). Amsterdam: John Benjamins.

Green, D. (2005). The neurocognition of recovery patterns in bilingual aphasics. In J. Kroll \& A. M. B. deGroot (Eds.), Handbook of bilingualism: Psycholinguistic approaches (pp. 516-530). London: Oxford University Press.

*Green, A., Schweda-Nicholson, N., Vaid, J., White, N., \& Steiner, R. (1990). Hemispheric involvement in shadowing vs. interpretation: A time-sharing study of simultaneous interpreters with matched bilingual and monolingual controls. Brain and Language, 39, 107-133.

Grosjean, F. (1989). Neurolinguists beware! The bilingual is not two monolinguals in one person. Brain and Language, 36, 3-15.

Grosjean, F., Li, P., Munte, T., \& Rodriguez-Fornells, A. (2003). Neuroimaging bilinguals: When the neurosciences meet the language sciences. Bilingualism: Language and Cognition, 6, 159-165.

*Hall, D. G., \& Lambert, W. E. (1988). French immersion and hemispheric language processing: A dual-task study. Canadian Journal of Behavioural Science, 20, 1-14.

Halsband, U. (2006). Bilingual and multilingual language processing. Journal of Physiology Paris, 99, 355-369.

*Hatta, V., 1982. Different hemisphere function in bilinguals and monolinguals. Unpublished manuscript. Japan: Kyoto University.

*Hausmann, M., Durmusoglu, G., Yazgan, Y., \& Güntürkün, O. (2004). Evidence for reduced hemispheric asymmetries in non-verbal functions in bilinguals. Journal of Neurolinguistics, 17(4), 285-299.

Hedges, L., \& Becker, B. (1986). Statistical methods in the meta-analysis of research on gender differences. In J. Hyde \& M. C. Linn (Eds.), The psychol- ogy of gender: Advances through meta-analysis. Baltimore: Johns Hopkins University Press.

Hedges, L., \& Olkin, I. (1985). Statistical methods for meta-analysis. Orlando, FL: Academic Press.

Hellige, J., \& Sergent, J. (1986). Role of task factors in visual field asymmetries. Brain and Cognition, 5, 200-222.

Hernandez, A., Martinez, A., \& Kohnert, K. (2000). In search of the language switch: An fMRI study of picture naming in Spanish-English bilinguals. Brain and Language, 73, 421-431.

Hickok, G., \& Poeppel, D. (2004). Dorsal and ventral streams: A framework for understanding aspects of the functional anatomy of language. Cognition, 92, 67-99.

*Hoosain, R., \& Shiu, L. (1989). Cerebral lateralization of Chinese-English bilingual functions. Neuropsychologia, 27(5), 705-712.

Hull, R., \& Vaid, J. (2005). Clearing the cobwebs from the study of the bilingual brain: Toward converging evidence-Laterality ERP. In J. Kroll \& A. M. B. deGroot (Eds.), Handbook of bilingualism: Psycholinguistic approaches (pp. 481-496). London: Oxford University Press.

Hull, R., \& Vaid, J. (2006). Laterality and language experience. Laterality, 11 , 436-464.

Hunter, J., \& Schmidt, F. (1990). Methods of meta-analysis: Correcting error and bias in research findings. Newbury Park, CA: Sage.

Illes, J., Francis, W. S., Desmond, J. E., Gabrieli, J. D. E., Glover, G. H., Poldrack, R., et al. (1999). Convergent cortical representation of semantic processing in bilinguals. Brain and Language, 70, 347-363.

Indefrey, P. (2006). A meta-analysis of hemodynamic studies on first and second language processing: Which suggested differences can we trust and what do they mean? In M. Gulberg \& P. Indefrey (Eds.), Cognitive neuroscience of second language acquisition (pp. 279-304). Malden, MA: Blackwell.

*Ip, K., \& Hoosain, R. (1993). Dichotic listening of Chinese and English words. Psychologia, 36, 140-143

*Jin, Y. (1988). Effects of word orthography and concreteness on cerebral hemispheric asymmetry in Korean bilinguals. Unpublished doctoral dissertation. Gainesville: The University of Florida.

Johnson, B. (1993). DSTAT 1.10: Software for the meta-analytic review of research literatures [computer software and manual]. Hillsdale, $\mathrm{NJ}$ : Lawrence Erlbaum Associates.

Johnson, J., \& Newport, E. (1989). Critical period effects in second language learning: The influence of maturational state on the acquisition of English as a second language. Cognitive Psychology, 21, 60-99.

-Judd, J. (1986). The effects of task difficulty and learning environment on the processing of verbal input as measured by the dichotic listening paradigm. Unpublished master's thesis. Provo, UT: Brigham Young University.

*Kang, M. (1984). The function of hemispheric lateralization in second language processing. Unpublished doctoral dissertation. Bloomington: Indiana University.

*Ke, C. (1992). Dichotic listening with Chinese and English tasks. Journal of Psycholinguistic Research, 21, 463-471.

*Kilborn, K., 2002. Hemispheric asymmetries in bilingual lexical processing. Unpublished manuscript. Scotland, UK: The University of Glasgow.

Kim, K., Relkin, N., Lee, K., \& Hirsch, J. (1997). Distinct cortical areas associated with native and second languages. Nature, 388, 171-174.

Klein, D., Milner, B., Zatorre, R., Zhao, V., \& Nikelski, J. (1999). Cerebral organization in bilinguals: A PET study of Chinese-English verb generation. Neuroreport, 10, 2841-2846.

Kosaka, B., Hiscock, M., Strauss, E., Wada, J., \& Purves, S. (1993). Dual task performance by patients with left or right speech dominance as determined by carotid amytal tests. Neuropsychologia, 31, 127-136.

-Kotik, B. (1975). Investigation of speech lateralization in multilinguals. Unpublished master's thesis. Russia: Moscow State University.

Kroll, J., \& Stewart, E. (1994). Category interference in translation and picture naming: Evidence for asymmetric connections between bilingual memory representations. Journal of Memory and Language, 33, 149-174.

Lenneberg, E. (1969). The neurobiology of language: Practical applications. In Paper presented at the annual meeting of the Orton Society.

Lipsey, M., \& Wilson, D. (2001). Practical meta-analysis. London: Sage.

Long, M. (1990). Maturational constraints on language development. Studies in Second Language Acquisition, 12, 251-285. 
-Lubow, R., Tsal, Y., Mirkin, A., \& Mazliah, G. (1994). English and Hebrew letter report by English- and Hebrew-reading subjects: Evidence for stimulus control, not hemispheric asymmetry. Brain and Cognition, 25, 34-51.

*Mägiste, E. (1987). Changes in the lateralization pattern of two immigrant groups in Sweden. In Y. Kim \& W. Gudykunst (Eds.), Cross-cultural adaptation: Current approaches (pp. 233-251). London: Sage.

*Mägiste, E. (1989). Conjugate lateral eye-movements in response to verbal, spatial, and emotional tasks. Investigaciones Psicologicas, 7, 69-77.

*Manga, D., \& Sanchez, M. (1989). Bilinguismo escolar y lateralizacion cerebral. Investigaciones Psicologicas, 7, 55-67.

-McClung, B. J. B. (1981). Lateralization of a second language by monolingual and bilingual subjects as determined by dichotic listening. Unpublished doctoral dissertation. Detroit, MI: Wayne State University.

Mechelli, A., Crinion, J., Noppeney, U., O’Doherty, J., Asburner, J., Frackowiak, R., et al. (2004). Structural plasticity in the bilingual brain. Nature, 431, 757.

Meuter, R. (2005). Language selection in bilinguals. In J. Kroll \& A. M. B. deGroot (Eds.), Handbook of bilingualism: Psycholinguistic approaches (pp. 349-370). London: Oxford University Press.

Neville, H., Coffey, S., Lawson, D., Fischer, A., Emmorey, K., \& Bellugi, U. (1997). Neural systems mediating American sign language: Effects of sensory experience and age of acquisition. Brain and Language, 47, 285308.

Newman, A., Izvorski, R., Davis, L., Neville, H., \& Ullman, M. (1999). Distinct electrophysiological patterns in the processing of regular and irregular verbs. Journal of Cognitive Neuroscience, S4.

Obler, L. (1981). Right hemisphere participation in second language acquisition. In K. Diller (Ed.), Individual differences and universals in language learning aptitude (pp. 53-64). Rowley, MA: Newbury.

Obler, L., Zatorre, R., Galloway, L., \& Vaid, J. (1982). Cerebral lateralization in bilinguals: Methodological issues. Brain and Language, 15, 40-54.

-Orbach, J. (1967). Differential recognition of Hebrew and English words in right and left visual fields as a function of cerebral dominance and reading habits. Neuropsychologia, 5, 127-134.

Paradis, M. (2000). Generalizable outcomes of bilingual aphasia research. Folia Phoniatrica Logopaedica, 52, 54-64.

Paradis, M. (2003). The bilingual Loch Ness Monster raises its non-asymmetric head again - Or, why bother with such cumbersome notions as validity and reliability? Brain and Language, 87, 441-448. Comments on Evans et al. (2002).

Pavlenko, A., \& Jarvis, S. (2002). Bidirectional transfer. Applied Linguistics, 23(2), 190-214.

Perani, D., Dehaene, S., Grassi, F., Cohen, S., Cappa, S., Dupoux, et al. (1996). Brain processing of native and foreign languages. Neuroreport, 7(15), 2439-2444

*Persinger, M., Chellew-Belanger, G., \& Tiller, S. (2002). Bilingual men but not women display less left ear but not right ear accuracy during dichotic listening compared to monolinguals. International Journal of Neuroscience, $112,55-63$

Poeppel, D., \& Hickok, G. (2004). Towards a new functional anatomy of language. Cognition, 92, 1-12.

Raichle, M. E., Fiez, J. A., Videen, T. O., McCloud, A. K., Pardo, J. V., MacLeod, A. K., et al. (1994). Practice-related changes in human brain functional anatomy during nonmotor learning. Cerebral Cortex, 4, 8-26.

*Rastatter, M., \& Scukanec, G. (1990). Evidence for hemispheric specialization of lexical distinctions in bilingual Chinese-Mandarin speakers. Cortex, 26 , 423-432.

Rosenthal, R. (1991). Meta-analytic procedures for social research. London: Sage.

Rosenthal, R., \& DiMatteo, M. (2001). Meta-analysis: Recent developments in quantitative methods for literature reviews. Annual Review of Psychology, $52,59-82$.

*Rupp, J. (1980). Cerebral language dominance in Vietnamese-English bilingual children. Unpublished doctoral dissertation. Albuquerque: The University of New Mexico.

*Sakhuja, V. (1990). Hemisphericity and cognitive concomitance. Unpublished doctoral dissertation. India: Psychology Department, University of Delhi.

Schneiderman, E. (1983). Leaning to the right: Some thoughts on hemisphere involvement in language acquisition. In J. Vaid (Ed.), Language processing in bilinguals: Psycholinguistic and neuropsychological perspectives (pp. 233-251). Hillsdale, NJ: Lawrence Erlbaum Associates.

Sebastian-Galles, N., \& Bosch, L. (2005). Phonology and bilingualism. In J. Kroll \& A. M. B. deGroot (Eds.), Handbook of bilingualism: Psycholinguistic approaches (pp. 68-87). London: Oxford University Press.

Segalowitz, S. (1986). Validity and reliability of noninvasive lateralization measures. Child Neuropsychology, 1, 191-208.

Segalowitz, S., \& Bryden, M. P. (1983). Individual differences in hemispheric representation of language. In S. J. Segalowitz (Ed.), Language functions and brain organization (pp. 341-372). New York: Academic Press.

*Sewell, D., \& Panou, L. (1983). Visual field asymmetries for verbal and dot localization tasks in monolingual and bilingual subjects. Brain and Language, 18, 28-34.

Seymour, P., Aro, M., \& Erskine, J. (2003). Foundation literacy acquisition in European orthographies. British Journal of Psychology, 94, 143-174.

*Shanon, B. (1982). Lateralization effects in the perception of Hebrew and English words. Brain and Language, 17, 107-123.

*Singh, M. (1990). Lateralized interference in concurrent manual activity: Influence of age in children. International Journal of Neuroscience, 50, 55-58.

-Soares, C. (1982). Converging evidence for left hemisphere language lateralization in bilinguals. Neuropsychologia, 20(6), 653-659.

*Soares, C. (1984). Left-hemisphere language lateralization in bilinguals: Use of the concurrent activities paradigm. Brain and Language, 23, 86-96.

-Soares, C., \& Grosjean, F. (1981). Left hemisphere language lateralization in bilinguals and monolinguals. Perception and Psychophysics, 29, 599-604.

*Spiller-Bosatra, E., Daro, V., Fabbro, F., \& Bosatra, A. (1990). Audiophonological and neuropsychological aspects of simultaneous interpretation. Scandinavian Audiology, 19, 81-87.

*Starck, R., Genesee, F., Lambert, W., \& Seitz, M. (1977). Multiple language experience and the development of cerebral dominance. In S. Segalowitz \& F. Gruber (Eds.), Language development and neurobiological theory (pp. 47-55). New York: Academic Press.

*Thomas, H. (1987). Effects of different acoustic characteristics in language processing: A dichotic listening study of Mandarin-English speakers. Unpublished doctoral dissertation. Bloomington: Indiana University.

Ullman, M. (2001). The neural basis of lexicon and grammar in first and second language: The declarative/procedural model. Bilingualism, 4(2), 105-122.

Ullman, M., Bergida, R., \& O'Craven, K. (1997). Distinct fMRI activation patterns for regular and irregular past tense. Neurolmage, 5, S549.

*Vaid, J. (1979). Visual field asymmetries in bilinguals on a bimodal rhyming task. Unpublished manuscript. Montreal, Quebec, Canada: McGill University.

*Vaid, J. (1980a). Visual field asymmetries for bimodal rhyme judgments in English/French early and late bilinguals and English monolinguals. Unpublished manuscript. Montreal, Quebec, Canada: McGill University.

*Vaid, J. (1980). Visual field asymmetries in bilinguals and monolinguals on a spatial Stroop task. In Paper presented at the annual meeting of BABBLE.

*Vaid, J. (1981a). Visual field asymmetries for rhyme and syntactic category judgments for visually presented stimuli. Unpublished manuscript. Montreal, Quebec, Canada: McGill University.

*Vaid, J. (1981b). Visual field asymmetries for rhyme judgments for visually presented stimuli. Unpublished manuscript. Montreal, Quebec, Canada: McGill University.

Vaid, J. (1983). Bilingualism and brain lateralization. In S. Segalowitz (Ed.), Language functions and brain organization (pp. 315-339). New York: Academic Press.

*Vaid, J. (1984). Visual, phonetic, and semantic processing in early and late bilinguals. In M. Paradis \& Y. Lebrun (Eds.), Early bilingualism and child development (pp. 175-191). Lisse: Swets \& Zeitlinger.

*Vaid, J. (1984b). Rhyme and semantic category judgments in English/French early bilinguals and French monolinguals. Unpublished manuscript. Montreal, Quebec, Canada: McGill University.

*Vaid, J. (1987). Visual field asymmetries for rhyme and syntactic category judgments in monolinguals and fluent early and late bilinguals. Brain and Language, 30, 263-277.

*Vaid, J. (1988). Asymmetries in tachistoscopic word recognition: Scanning effects re-examined. International Journal of Neuroscience, 42, 253 258 . 
*Vaid, J. (1999). Laterality of semantic category judgments in bilinguals. Unpublished manuscript. College Station: Texas A\&M University.

*Vaid, J. (2001). Lateralization of counting skill in bilinguals: A dual task study. Brain and Cognition, 46(1-2), 14.

*Vaid, J. (2003). Cerebral laterality for gender classification of proper names: Evidence from bilinguals. Unpublished manuscript. College Station: Texas A\&M University.

Vaid, J. (in press). The bilingual brain: What's right? What's left? In J. Altarriba, R. Heredia (Eds.), An introduction to bilingualism: Principles and processes. Mahwah, NJ: Erlbaum.

*Vaid, J., \& Frenck-Mestre, C. (1990). Language recognition in skilled bilinguals. Unpublished manuscript. College Station: Texas A\&M University.

*Vaid, J., \& Frenck-Mestre, C. (2002). Do orthographic cues aid language recognition? A laterality study with French-English bilinguals. Brain and Language, 82, 47-53.

Vaid, J., \& Genesee, F. (1980). Neuropsychological approaches to bilingualism: A critical review. Canadian Journal of Psychology, 34, 417-445.

Vaid, J., \& Hall, D. G. (1991). Neuropsychological perspectives on bilingualism: Right, left and center. In A. Reynolds (Ed.), Bilingualism, multiculturalism and second language learning: The McGill conference in honour of Wallace E. Lambert (pp. 81-112). Hillsdale, NJ: Lawrence Erlbaum Associates.

Vaid, J., \& Hull, R. (2002). Re-envisioning the bilingual brain using functional neuroimaging: Methodological and interpretive issues. In F. Fabbro (Ed.), Advances in the neurolinguistics of bilingualism: A festschrift for Michel Paradis (pp. 315-355). Udine, Italy: Forum.

*Vaid, J., \& Lambert, W. (1979). Differential cerebral involvement in the cognitive functioning of bilinguals. Brain and Language, 8, 92-110.
*Vaid, J., \& Park, K. (1997). Hemispheric asymmetries in reading Korean: Task matters. Brain and Language, 58, 115-124.

Vingerhoets, G., Van Borsel, J., Tesink, C., van den Noort, M., Deblaere, K., Seurinck, R., et al. (2003). Multilingualism: An fMRI study. NeuroImage, 20, 2181-2196.

Voyer, D. (1996). On the magnitude of laterality effects and sex differences in functional lateralities. Laterality, 1(1), 51-83.

*Voyer, D., Cormier, P., \& Boudreau, V. (2002). Language lateralization in English-French bilinguals and monolingual speakers. Unpublished manuscript. Canada: The University of New Brunswick.

Wartenburger, I., Heekeren, H. R., Abutalebi, J., Cappa, S. F., Villringer, A., \& Perani, D. (2003). Early setting of grammatical processing in the bilingual brain. Neuron, 37, 159-170.

*Wesche, M., \& Schneiderman, E. (1982). Language lateralization in adult bilinguals. Studies in Second Language Acquisition, 4(2), 153-169.

*Winfield, F. (1984). Cerebral lateralization, preferred cognitive mode and reading achievement in American Indian bilingual children. Unpublished thesis. Flagstaff: Northern Arizona University.

Wood, W., \& Quinn, J. (2003). Forewarned and forearmed? Two meta-analytic syntheses of forewarnings of influence appeals. Psychological Bulletin, 129(1), 119-138.

*Wuillemin, D., Richardson, B., \& Lynch, J. (1994). Right hemisphere involvement in processing later-learned languages in multilinguals. Brain and Language, 46, 620-636.

*Yoshizaki, K., \& Hatta, V. (1987). Shift of visual field advantage by learning experience of foreign words. Neuropsychologia, 25(3), 589-592.

Zatorre, R. (1989). On the representation of multiple languages in the brain: Old problems and new directions. Brain and Language, 36, 127-147. 